\title{
Disease Tolerance and Immunity in Host Protection Against Infection
}

\author{
Miguel P. Soares*, Luis Teixeira, Luis F. Moita \\ Instituto Gulbenkian de Ciência, Rua da Quinta Grande, 6, 2780-156 Oeiras, Portugal \\ *Correspondence to MPS: mpsoares@igc.gulbenkian.pt
}

\begin{abstract}
The immune system has most likely evolved to limit the negative impact exerted by pathogens on host homeostasis. This defense strategy relies on the concerted action of innate and adaptive components of the immune system, which sense and target pathogens for containment, destruction or expulsion. Resistance to infection refers to these immune functions, which reduce the pathogen load of an infected host as the means to preserve homeostasis. Immune-driven resistance to infection is coupled to an additional, and arguably as important, defense strategy that limits the extent of dysfunction imposed to host parenchyma tissues during infection, without exerting a direct negative impact on pathogens. This defense strategy, called disease tolerance, relies on tissue damage control mechanisms that prevent the deleterious effects of pathogens, while uncoupling immunedriven resistance mechanisms from immunopathology and disease. Here we provide a unifying view of resistance and disease tolerance within the framework of immunity to infection.
\end{abstract}

\section{INTRODUCTION}

The pathological outcome of infection is largely determined by the degree of metabolic dysfunction and damage inflicted upon the host's parenchyma tissues ${ }^{1,2}$. Clinical signs and symptoms of infectious diseases emerge as host homeostasis [G] becomes compromised due to tissue dysfunction and damage ${ }^{1-3}$. Current understanding of this pathological process is limited, presumably impairing the ability to treat infectious diseases that remain associated with high human morbidity and mortality ${ }^{4}$.

Immunity provides protection against disease, in general, and particularly against infectious diseases ${ }^{4}$. This is achieved by virtue of immune-driven resistance mechanisms that expel, contain, or kill pathogens as the means to preserve host homeostasis. Therapeutic approaches based on the induction of such immune-driven resistance mechanisms, such as vaccination, have proven highly protective against a broad range of infectious diseases ${ }^{4}$. This is also the case for anti-microbial agents, like antibiotics, that 
functionally mimic resistance mechanisms containing or killing pathogens ${ }^{4}$. The overwhelming success of these therapeutic approaches has likely contributed to the perception that resistance mechanisms are the only relevant defense strategy against infectious diseases. This notion has been challenged over the past years by the (re)discovery of disease tolerance ${ }^{5,6}$. This evolutionarily conserved host defense strategy, which was first described in plants ${ }^{7,8}$, is fully operational in flies ${ }^{9,10}$ and mammals, including rodents $^{11,12}$ and humans ${ }^{13}$, where it preserves host homeostasis in response to viral ${ }^{14,15}$, bacterial $^{15-18}$, fungal ${ }^{19}$ and protozoan ${ }^{11,13,20,21}$ infections. In contrast to resistance to infection, disease tolerance does not exert a direct negative effect on these pathogens ${ }^{6}$.

Revealed through the recognition that variation in disease severity can occur at a population level without a direct correlation to pathogen load, disease tolerance is now widely studied mechanistically, using experimental models of infection in which the relationship between host health and pathogen load can be established at an individual level (Box 2). Here we review the cellular and molecular mechanisms conferring disease tolerance to infection and explore the impact exerted by bona fide immunity on those mechanisms. We also bring to light how the establishment of symbiotic interactions with microbes and their regulation by specific components of innate and adaptive immunity impact on disease tolerance to infection (see also ${ }^{22}$ ). Finally, we put forward that the mechanisms underlying disease tolerance to infection can be targeted therapeutically against infectious diseases.

\section{TISSUE DAMAGE CONTROL}

The mechanisms underlying disease tolerance remain poorly understood, but appear to revolve around a number of evolutionarily conserved stress and damage responses conferring tissue damage control in the infected host ${ }^{1-3}$ (Figure 1). These stress and damage responses sustain the functional outputs of host parenchyma cells under different forms of stress and damage imposed either directly by pathogens, i.e. virulence, or indirectly by host immune-driven resistance mechanisms, i.e. immunopathology ${ }^{1,3}$ [G]. Stress and damage responses provide metabolic adaptation while repairing damage to cellular metabolites, macromolecules and/or organelles, as the means to preserve core cellular functions, often to the detriment of accessory ones ${ }^{1,3}$. When these responses fail per se to sustain the functional outputs of parenchyma tissues the default program becomes programmed cell death (Figure 1). This is coupled to the induction of cellular and tissue regenerative responses that restore the functional output of damaged parenchyma tissues ${ }^{3,23}$. As discussed in further detail below, different types of infection impose distinct forms of stress and damage to host parenchyma cells, suggesting that tissue damage control mechanisms 
might act in a somewhat pathogen class-specific manner that reflects these differences such as to effectively establish disease tolerance against diverse types of infection ${ }^{6}$. While there are clearly parallels between the protective effect exerted by tissue damage control mechanisms in the context of infectious and non-infectious diseases ${ }^{1,6,24}$, we shall restrict our discussion here to infectious diseases.

\section{STRESS RESPONSES IN TISSUE DAMAGE CONTROL}

Stress responses are triggered through the engagement of specific sensors that continuously monitor different physiological parameters under homeostatic regulation such as temperature, $\mathrm{O}_{2}, \mathrm{pH}$, osmolarity, glucose and $\mathrm{ATP}^{1-3}$. When these parameters change beyond a certain threshold, "stress sensors" set off signal transduction pathways that alert cells for a possible disruption of homeostasis ${ }^{1-3}$. The ensuing stress responses provide metabolic adaptation in host cells, conferring tissue damage control and disease tolerance to infection $^{1-3}$ (Figure 1\&2). While recognition of pathogens via pattern recognition receptor (PRR) can contribute to tissue damage control and to the establishment of disease tolerance ${ }^{25}$, we shall not address it in further detail herein as this has been covered in detail elsewhere ${ }^{24,26}$. Instead we shall highlight a number of bona fide stress responses involved in tissue damage control mechanisms that contribute to the establishment of disease tolerance to infection ${ }^{1-3}$.

Oxidative stress. The oxidative stress response orchestrated by the transcription factor, nuclear factor E2-related factor-2 $(\mathrm{NRF} 2)^{27,}{ }^{28}$ (Figure 2) can contribute critically to the establishment of disease tolerance to infection, as demonstrated for malaria ${ }^{20,29}$. Briefly, the blood stage of Plasmodium infection is associated with hemolysis and hence with generation of extracellular hemoglobin ${ }^{20,21,29,30}$. Upon oxidation, extracellular hemoglobin releases its prosthetic heme groups, which act as catalysts in the production of reactive oxygen species (ROS) and nitrogen species (RNS). This can lead to oxidative stress and cellular damage in host parenchyma tissues ${ }^{12,13,16,31}$, driving the pathogenesis of severe forms of malaria ${ }^{30}$. Sickle hemoglobin, a genetic polymorphism in the $\beta$ chain hemoglobin gene naturally selected through human evolution, confers disease tolerance to malaria ${ }^{20,32}$, via a mechanism that counters the pathogenic effects of labile heme via the activation of NRF2 $2^{20}$ 30,33 . While NRF2 is also protective against polymicrobial ${ }^{34}$ and Staphylococcus aureus ${ }^{35}$ infections, it is not clear whether this is due to the establishment of disease tolerance but the two following observations suggest that it is so: i) NRF2 polarizes macrophage responses towards the promotion of tissue damage control and disease tolerance ${ }^{36,37}$, and ii) NRF2 induces mitochondrial biogenesis in parenchyma cells ${ }^{35}$, which is likely to contribute to tissue damage control and disease tolerance to infections. 
Hypoxia. Infections can be associated with local or systemic decrease in $\mathrm{O}_{2}$ supply to host cells, a condition known as hypoxia. This is sensed and countered by host cells via a stress response controlled by the hypoxia inducible factor (HIF) family of transcription factors ${ }^{38}$ (Figure 2). Whether HIF activation in parenchyma cells contributes to the establishment of disease tolerance to infection has not been established, but HIF activation in macrophages, which shifts ATP production via anaerobic glycolytic metabolism, modulates macrophage polarization ${ }^{39,40}$ towards an effector response that promotes tissue damage control and disease tolerance to infection, as illustrated for Helicobacter pylori infection in mice ${ }^{41}$.

Metabolic stress. Stress sensors monitor variations in the relative concentration of essential metabolites such as for example ATP and glucose ${ }^{1,3}$. These variations activate stress responses that adjust host cellular metabolism to the relative availability of the metabolites sensed. Presumably metabolic stress responses confer tissue damage control and contribute to the establishment of disease tolerance to different types of infection (Figure 2). In strong support of this notion is the recent finding that stress responses that adjust host glucose metabolism confer tissue damage control and establish disease tolerance to viral and bacterial infections in mice ${ }^{42}$.

Osmotic stress. Osmotic stress, associated with systemic infections ${ }^{43}$, is sensed and regulated by the infected host, via several mechanisms including the activation of nuclear factor of activated T cells 5 (NFAT5) ${ }^{44}$ (Figure 2). The osmotic stress response regulated by this transcription factor acts in a cytoprotective manner in parenchyma cells ${ }^{45}$, conferring tissue damage control in the kidney during systemic polymicrobial infections ${ }^{46}$ and likely in the heart during infection with coxsackievirus ${ }^{47}$ in mice (Figure 2).

\section{DAMAgE RESPONSES AND TISSUE DAMAGE CONTROL}

When stress associated with infection persists in strength and/or time, metabolic adaptation is no longer sufficient per se to preserve core cellular functions in parenchyma tissues and organs sustaining homeostasis. The ensuing damage inflicted to cellular metabolites, macromolecules, i.e. DNA, proteins and lipids, and organelles, activates specific damage responses that contribute to tissue damage control and to the establishment of disease tolerance to infection ${ }^{1-3}$ (Figure 1\&2).

Metabolite damage and extracellular release. Metabolite damage refers to modifications of metabolites that compromise their original function ${ }^{48}$, exerting pathologic effects, namely: i) impairing host cellular functions and ii) generating "toxic" products that catalyze inflammation, cellular stress and damage ${ }^{48}$. For example, modifications of soluble 
metabolites that promote phase transition into crystals, are sensed by PRR and trigger inflammatory responses that are deleterious to the host ${ }^{49}$. It follows that limiting the proinflammatory effects of metabolite damage can promote disease tolerance to infection (Figure 2).

Lipid damage. Lipid peroxidation can impair cellular membrane function, eventually leading to release of intracellular content ${ }^{50}$ (Figure 2). Moreover, sensing of lipid peroxidation products by the PRR Toll-like receptor 4 (TLR4), tissue damage, as demonstrated in the pathogenesis of Influenza virus infection ${ }^{51}$. Inhibition of lipid peroxidation by peroxidase 4 $(\mathrm{GPX} 4)^{28,52}$, relies on glutathione supply by a NRF2-regulated pathway involving, SLC7A11, which together with SLC3A2 encode the cystine/glutamate antiporter, GCLC and GCLM that encode the $\gamma$-glutamylcysteine synthetase ( $\gamma G C S$ ) and GSS that encodes the glutathione synthetase $\mathrm{e}^{28,52}$. Activation of the transcription factor NRF2 also counters lipid peroxidation via the expression of heme oxygenase-1 (HO-1) ${ }^{31}$ (Figure 2).

Proteoxic damage. Proteotoxic damage responses are aimed at repairing or eventually degrading unfolded nascent or mature proteins as a means to preserve essential cellular functions ${ }^{53,54}$. Proteotoxic damage responses can act as bona fide tissue damage control mechanisms, promoting the establishment of disease tolerance to bacterial infection ${ }^{53,55-58}$ (Figure 2). Of note, proteotoxic damage responses are also involved in the regulation of immune-driven resistance mechanisms against intracellular bacteria and viruses, some of which evolved to modulate these responses as a strategy to repress host resistance to infection $^{53} 59$

DNA damage. Infections are associated with DNA damage and the activation of DNA damage responses ${ }^{60}$ (Figure 2). This occurs most likely via a mechanism involving the Mre11/Rad50/Nbs1 complex, which activates for example ataxia-telangiectasia mutated (ATM) kinase, a master regulator of the double-stranded DNA damage response ${ }^{61}$. Activation of ATM confers tissue damage control and disease tolerance to polymicrobial infections in mice ${ }^{17}$, suggesting that DNA damage responses contribute to maintain both the genetic integrity and the functional outputs of parenchyma cells during infection.

Damage to cellular organelles. Autophagy is an evolutionary conserved damage response that supports cell function under stress conditions that elicit damage to cellular organelles (Figure 2) ${ }^{62}$. Autophagy regulates inflammatory responses and modulates disease tolerance to infections such as Sindbis virus ${ }^{63}, S$. aureus $^{64}$ and polymicrobial ${ }^{17}$ infections in mice. Of note, autophagy can act as a resistance mechanism against intracellular pathogens, i.e. xenophagy ${ }^{62}$. 


\section{Programmed Cell DeAth in tissue damage ContRol}

When stress and damage responses fail to preserve cellular functional outputs, the default response is, in most cases, programmed cell death (Figure 1). Different forms of stress and damage are associated with distinctive forms of programmed cell death and ensuing pathophysiological consequences ${ }^{65}$. For example, failure to resolve oxidative stress can lead necroptosis [G] ${ }^{66}$ while failure to repair lipid peroxidation induces ferroptosis [G] ${ }^{67}$. Irreparable DNA damage results in the induction of different programmed cell death pathways, including apoptosis ${ }^{68}$, regulated by the caspase family of cysteine proteases.

While programmed cell death can damage host parenchyma tissues and lead to organ dysfunction, this relationship is not always straightforward because programmed cell death is also part of a resistance mechanism against intracellular pathogens ${ }^{69}$. However, the trade-off of this resistance mechanism can be particularly high, depending on the relative capacity of different tissues to withstand cell loss without compromising tissue function and homeostasis $^{6}$. The pathophysiological relevance of this defense strategy is supported by the number of mechanisms deployed by intracellular pathogens to promote or inhibit host genetic programs controlling programmed cell death, presumably as a strategy to escape resistance to infection ${ }^{70}$.

Programmed cell death of infected cells is coupled to their immediate phagocytosis by bystander macrophages. Dendritic cells (DCs) also take up dying infected cells, processing and presenting pathogen-associated antigens to $\mathrm{CD}^{+} \mathrm{T}$ helper $\left(\mathrm{T}_{\mathrm{H}}\right)$ or $\mathrm{CD} 8^{+}$cytotoxic $\mathrm{T}$ lymphocytes (CTLs) and eliciting antigen-specific adaptive immune responses that target and kill the remaining reservoir of infected cells.

Clearance of damaged and dying cells is associated with macrophage polarization towards the production of cytokines and pro-resolving lipid mediators ${ }^{71}$ including IL-10 and 15-Deoxy- $\Delta$-prostaglandin $\mathrm{J}_{2}\left(15 \mathrm{~d}-\mathrm{PGJ}_{2}\right)$, respectively, which induce the expression $\mathrm{HO}-1^{72}$, ${ }^{73}$. This macrophage response is also associated with the production of growth factors, such as platelet-derived growth factor (PDGF) and cytokines, such as transforming growth factor$\beta 1$ (TGF $\beta 1$ ), which can act directly on parenchyma cells to promote tissue regeneration, orchestrating yet another layer of tissue damage control ${ }^{23}$.

While some tissue damage control mechanisms act in cell-autonomous manner, others appear more to rely on bystander innate and adaptive immune cells. As discussed in the next section, tissue resident leukocytes play a predominant role in this non-cell autonomous establishment of disease tolerance to infection. 


\section{IMMUNE REGULATION OF TISSUE DAMAGE CONTROL}

In this section we illustrate how tissue-resident macrophages, innate lymphoid cells (ILCs) and regulatory $\mathrm{T}\left(\mathrm{T}_{\mathrm{REG}}\right)$ cells impact on tissue damage control mechanisms, and thereby contribute in a non cell-autonomous manner to the establishment of disease tolerance to infection (Figure 3).

Macrophages. Tissue resident macrophages express high levels of PRR and a variety of other receptors that sense pathogen associated molecular patterns (PAMP), damage associated molecular patterns (DAMP) and other alarmins [G] including cytokines released from damaged cells, which alert for disruption of homeostasis ${ }^{74,75}$ (Figure 3a). These cytokines include IL-1a, IL-18 and IL-33, which polarize macrophages towards tissue healing regenerative responses, and as discussed in the previous section, contribute to the establishment of disease tolerance to infection (Figure 3a). Signaling via the aryl hydrocarbon receptor $(\mathrm{AhR})$ a ligand-dependent transcription factor that senses exogenous environmental toxins and endogenous ligands, can also polarize macrophages towards the establishment of disease tolerance to bacterial infections ${ }^{18}$.

Macrophage anti-microbial responses are also associated with the expression of genes that can promote tissue damage control in parenchyma cells, for example, inducible nitric oxide synthase (NOS2), HO-1 and cystathionine $\beta$-synthase (CBS), which generate nitric oxide (NO), $\mathrm{CO}$ and hydrogen sulfide $\left(\mathrm{H}_{2} \mathrm{~S}\right)$, respectively (Figure 3a). These gasotransmitters ${ }^{76}$ can diffuse across cellular membranes and drive metabolic adaptation in microbes $^{77}$ as well as in parenchyma cells ${ }^{76,78}$, supporting tissue damage control and disease tolerance to infection (Figure 3a). Cytokines produced during these responses exert a dual role, for example, tumor necrosis factor (TNF) can trigger programmed cell death while also activating pro-survival responses via activation of the nuclear factor kappa B (NF$\kappa \mathrm{B})$ family of transcription factors ${ }^{79}$ (Figure $3 a$ ).

Phagocytosis is also associated with the production of ROS by macrophages, supporting stem cell division and differentiation towards tissue healing and regeneration ${ }^{80}$. The mechanism via which this occurs involves the repression $\mathrm{Nrf} 2^{81}$, revealing a tight integration of stress and damage responses with subsequent tissue repair and regeneration programs restoring host homeostasis ${ }^{23,80,82}$ (Figure 1,2). How these apparently conflicting activities exerted by cytokines such as TNF or ROS are resolved remains to be understood ${ }^{23}$. This suggests nevertheless, that anti-microbial macrophage responses have built-in feedback loops promoting tissue damage control. 
Innate lymphoid cells. All three classes of ILCs, i.e. ILC1, ILC2 and ILC3, play a critical role in sensing tissue dysfunction and damage, orchestrating tissue damage control responses ${ }^{83}$, which contribute to disease tolerance to viral ${ }^{84}$ and helminthic ${ }^{85}$ infections as well during intestinal inflammation ${ }^{86}$. For example, ILC2 can sense alarmins, such as IL-33, and promote tissue damage control in epithelia, via a mechanism that involves the production of the EGFlike factor amphiregulin ${ }^{83,84,86}$ (Figure $3 b$ ). Engagement of natural cytotoxicity receptors (NCRs) in ILC3 can sense ligands expressed by pathogens as well as self-ligands upregulated in response to cellular stress ${ }^{83}$, resulting in the production of IL-22, an IL-10 family member that promotes epithelia repair and regeneration ${ }^{87}$ (Figure $3 b$ ).

Regulatory $T$ cells $\left(T_{R E G}\right)$. Natural loss of function mutations in the $T_{R E G}$ cell lineage commitment transcription factor forkhead box P3 (Foxp3) are associated with development of severe immunopathology, in mice and in humans ${ }^{88}$. This suggests that $T_{\text {REG }}$ cells contribute to tissue damage control and presumably therefore regulate the establishment of disease tolerance to infection. The physiological functions assigned to $T_{\text {REG }}$ cells have long been related, almost exclusively, to their capacity to restrain adaptive and to a lesser extent innate immune responses ${ }^{88}$. More recently however, tissue-resident $T_{R E G}$ cells were show to promote tissue damage control mechanisms ${ }^{89-91}$ that confer disease tolerance to Influenza virus infection ${ }^{90}$. This protective effect involves signaling through alarmins, such as IL-18 or IL-33, that elicit the production and secretion of amphiregulin ${ }^{15,89,90}$ by tissue-resident $T_{\text {REG }}$ cells ${ }^{89-91}$ (Figure $3 c$ ). Whether tissue-resident $\mathrm{T}_{\text {REG }}$ cells protect parenchyma cells via direct interaction, or indirectly through immunoregulatory mechanisms involving tissue-resident macrophages or ILCs has not been established (Figure 3c).

Stress and damage responses in immune cells. When exposed to different forms of infection-associated stress, tissue-resident macrophages, ILCs and $T_{\text {REG }}$ cells should activate the same stress and damage responses that operate in parenchyma cells to promote tissue damage control and disease tolerance to infection. For example, NRF2 activation in macrophages ${ }^{36}$ and $\mathrm{T}$ cells ${ }^{92}$ exerts immunoregulatory effects that promote tissue damage control. Furthermore, HIF1 $\alpha$ orchestrates a metabolic response in macrophages ${ }^{40,93}$ and shifts the balance between $T_{H} 17$ and $T_{R E G}$ cells ${ }^{94}$, modulating immune-driven resistance and possibly disease tolerance to infection. Activation of other stress and damage responses in tissue resident leukocytes is likely to exert similar effects, but this is yet to be determined experimentally. 


\section{PATHOGEN CLASS-SPECIFIC TISSUE DAMAGE CONTROL}

Multicellular organisms establish stable symbiotic interactions with a wide variety of microorganisms, which impact on different aspects of their physiology ${ }^{95}$, while modulating resistance as well as disease tolerance to pathogens $(\operatorname{Box} 3)^{22}$. Infection by viruses, bacteria, fungi, protozoan parasites or helminthes impose distinct forms of stress and damage to host parenchyma cells, driven by intrinsic differences in virulence mechanisms as well as by the countervailing host immune-driven resistance mechanisms elicited. Thus, tissue damage control mechanisms probably act in a pathogen-specific manner to confer disease tolerance to different types of infection, as demonstrated originally in flies ${ }^{96}$ and thereafter in mice ${ }^{6,97,98}$. In this section we discuss how immune-driven resistance to a different pathogen classes regulates tissue damage control mechanisms to establish disease tolerance to those classes of pathogens.

Intracellular pathogens. Host resistance to intracellular pathogens, such as viruses and certain bacteria, relies to a large extent on cytotoxic mechanisms driven by type 1 immunity and the subsequent immune targeting of infected cells for programmed cell death ${ }^{99}$. Most immune-driven mechanisms killing intracellular pathogens fall under the control of the $T_{H} 1$ signature cytokine IFN $\gamma$, which promotes tissue damage through various mechanisms, including i) macrophage polarization towards an anti-microbial response associated with the production of ROS and RNS, ii) activation of $C D 8^{+}$cytotoxic $T\left(T_{C}\right)$ cells and NK cells to kill infected cells via the perforin/granzyme B-dependent lytic pathway or via the ligation of surface death receptors and iii) B cell activation towards the production of cytolytic antibodies, targeting infected cells for complement and Fc receptor mediated cellular cytotoxicity (Figure 4a). Tissue damage control mechanisms countering type 1 immunopathology should not therefore rely on cytoprotection of infected cells, as this would compromise resistance to intracellular pathogens. Instead these tissue damage control mechanisms promote cell regeneration and tissue repair as the means to compensate for loss of parenchyma cells and to restore tissue homeostasis ${ }^{15,} 90$ (Figure 4a). The mechanisms via which this occurs are not fully established but are likely to be tissue specific $^{6}$ and to involve the production of epidermal growth factors (EGF) and platelet derived growth factor (PDGF) family members as well as cytokines such as transforming growth factor (TGF)- $\beta$, which promote stem cell proliferation and/or differentiation into parenchyma cells and thereby restore tissue integrity and function ${ }^{23}$ (Figure $4 a$ ).

Extracellular parasites. Resistance against extracellular metazoan parasites relies to a large extent on type 2 immunity $^{99}{ }^{100}$. Some of these parasites are damaging to host 
parenchyma tissues, presumably explaining why type 2 immunity promotes the activation of tissue damage control mechanisms that confer disease tolerance to infection ${ }^{101}$ (Figure $4 \mathrm{~b}$ ). Type 2 immunity targets pathogens primarily for containment or expulsion, likely based on the inherent failure of immune-driven cytotoxic molecules to kill large metazoan parasites such as helminths (Figure 4b). In addition, type 2 cytokines promote macrophage polarization towards granuloma formation, containing pathogens and depriving them from essential nutrients. This resistance mechanism, known as nutritional immunity [G], is perhaps best illustrated by the modulation of host iron metabolism as a strategy to deprive pathogens from this essential nutrient ${ }^{102}$. However, macrophage polarization by type 2 cytokines also deprives pathogen access to other essential nutrients such as arginine, depleted by arginase expression in macrophages ${ }^{103}$. Moreover, arginine depletion also controls sustained $T$ cell activation and as such limits immunopathology ${ }^{103}$. As a trade-off, nutritional immunity can impose metabolic stress to host parenchyma cells, activating stress responses that confer metabolic adaption and possibly tissue damage control and disease tolerance to extracellular parasites (Figure $4 b$ ).

Extracellular bacteria and fungi. Resistance to infections by extracellular bacteria and fungi relies to a large extent on $T_{H} 17$ immunity ${ }^{99}$. Most of the resistance mechanisms associated with $\mathrm{T}_{\mathrm{H}} 17$ immunity, including pathogen killing, are mediated via the recruitment and activation of neutrophils ${ }^{99}$. This is often associated with the development of immunopathology, as illustrated at epithelial barriers, where ROS and elastase produced by neutrophils can cause epithelial cell damage and compromise disease tolerance to pathogenic Gram-negative bacteria such as Burkholderia pseudomallei ${ }^{104}$ (Figure 4c). $\mathrm{T}_{H} 17$ immunopathology is counteracted by tissue damage control mechanisms that protect epithelial barriers against oxidative stress and damage as well by additional mechanisms promoting tissue repair and regeneration (Figure $4 c$ ). Some of these are regulated directly or indirectly by IL-22, produced by $\mathrm{T}_{\mathrm{H}} 17$ or by ILC3 cells and signaling via the IL22R expressed by epithelial cells ${ }^{84,86,105}$. Other cytokines produced by $T_{H} 17$ cells, including IL-17, can amplify this protective response, synergizing with fibroblast growth factor (FGF) 2 produced by $T_{R E G}$ cells to promote tissue damage control at epithelial barriers ${ }^{106}$ (Figure $4 c$ ).

\section{DiseASE TOLERANCE TO CO-INFECTIONS}

Pathogen class-specific tissue damage control mechanisms are particularly relevant in the context of co-infections, as illustrated for bacterial pneumonia following influenza virus infection ${ }^{107}$. This is probably also the case for co-infections by rhinovirus, adenovirus or parainfluenza and Streptococcus pneumoniae as well as for Haemophilus influenza and 
Moraxella catarrhalis co-infections ${ }^{107}$. Several mechanisms may contribute to worsen the clinical outcome of these co-infections in that viral infections can compromise immune-driven resistance ${ }^{97}$ or tissue damage control ${ }^{15}$ mechanisms against bacterial co-infections, as illustrated for influenza virus and Legionella pneumophila co-infections ${ }^{97} 15$. The mechanisms via which viruses compromises disease tolerance to secondary bacterial infections are probably multi factorial and have been associated with deregulated production of EGF-like factors, such as amphiregulin ${ }^{15}$, presumably by lung-resident ILCs ${ }^{84}$ and $\mathrm{T}_{\mathrm{REG}}{ }^{90}$.

Immune-driven resistance mechanisms targeting viruses can also promote, rather than impair, disease tolerance to bacterial infections. For example, regulation of IL-1 $\beta$-induced inflammation by type I IFN prevents the lethality of systemic Streptococcus pyogenes infection, without affecting bacterial load ${ }^{108}$. In this case, signaling via type I IFN receptor in macrophages, DCs and neutrophils represses IL-1 $\beta$ transcription, through signal transducer and activator of transcription STAT1, and to a lesser extent STAT2, ultimately preventing the development of lethal IL-1 $\beta$-driven inflammation ${ }^{108}$.

Disease tolerance against one class of pathogens has also been shown to antagonize immune-driven resistance against other pathogen-classes. For example, the induction of HO-1 expression promotes disease tolerance to the blood stage of Plasmodium infection ${ }^{12}$, 20, 21, 30 but impairs resistance to Salmonella enterica subsp. enterica serovar Typhimurium co-infection ${ }^{98}$. This is thought to involve deregulated heme-driven mobilization of granulocytes from the bone marrow during Plasmodium infection with concomitant induction of $\mathrm{HO}-1$, reducing subsequent capacity of myeloid cells to generate ROS in response to $S$. Typhimurium $^{98}$. Of note, genetic confirmation of this mechanism requires further investigation.

\section{PHARMACOLOGICAL MODULATION OF DISEASE TOLERANCE}

In some cases, effective pathogen elimination by immune-driven resistance mechanisms fails to overcome the morbidity or mortality associated with infection. Moreover, current antimicrobial approaches also often fail to treat infectious diseases. In these cases, a rational pharmacological targeting of stress and damage responses controlling tissue damage control may act therapeutically through the establishment of disease tolerance to infection, as illustrated for pharmacological targeting of adenosine receptors ${ }^{109}$ or labile heme ${ }^{16}$, which establish disease tolerance to sepsis ${ }^{16,109}$ or malaria $^{20,21,30}$ in mice.

Several pharmacological strategies can be envisioned when targeting labile heme, as it accumulates in plasma during bloodstream infections. In some instances, administration of hemopexin, a plasma protein that binds avidly to labile heme and neutralizes its deleterious 
effects, confers tissue damage control and disease tolerance to sepsis in mice ${ }^{16}$. The pathological effects of labile heme can also be neutralized by gasotransmitters such as $\mathrm{CO}^{21}$ or $\mathrm{NO}^{29}$, which bind avidly to ferrous heme-iron $\left(\mathrm{Fe}^{2+}\right)$ in hemoproteins. In the case of $\mathrm{CO}$, this gasotransmitter blocks heme-iron oxidation $\left(\mathrm{Fe}^{2+}->\mathrm{Fe}^{3+}\right)$ and inhibits heme release from hemoproteins, preventing its accumulation in plasma during bloodstream infections ${ }^{20,} 21$. Remarkably, this is sufficient to confer protection against lethal forms of severe malaria in mice ${ }^{20,21,30}$. When used pharmacologically, NO also suppresses the pathogenesis of severe forms of malaria in mice, but this occurs via an indirect mechanism involving the activation of the transcription factor NRF2, the induction of $\mathrm{HO}-1$ expression and the downstream generation of $\mathrm{CO}$, which consequently establishes disease tolerance to malaria ${ }^{30,31}$. In addition from preventing heme release from extracellular hemoglobin, CO exerts cytoprotective effects ${ }^{110}$ that are likely to promote tissue damage control and disease tolerance to infection. Labile heme is a potent pro-oxidant and as such pharmacologic use of anti-oxidants such as $\mathrm{N}$-acetyl cysteine are efficient in preventing its pathogenic effects, conferring tissue damage control and disease tolerance to malaria ${ }^{12,}{ }^{13}$. Moreover, downstream events in the signaling transduction pathways driving heme cytotoxicity, e.g. sustained activation of c-Jun $\mathrm{N}$-terminal kinases, can also be targeted pharmacologically to promote recovery and survival through tissue damage control during malaria infection ${ }^{13}$.

Pharmacological targeting of metabolic stress responses such as the one regulated by AMPK, confers protection against the development of organ dysfunction associated with the pathogenesis of sepsis in mice ${ }^{111}$. This is not associated with overt modulation of the host pathogen load, suggesting that AMPK activation contributes to the establishment of disease tolerance to sepsis ${ }^{111}$. In further support of this notion, pharmacological AMPK activation in the brain is sufficient per se to confer protection against polymicrobial infections in mice ${ }^{112}$.

Another possible approach consists in targeting the inflammatory response to infection, without interfering with immune-driven resistant mechanisms. This can be achieved, for example via pharmacologic inhibition of topoisomerase 1 (Top1), suppressing the recruitment of RNA polymerase II (RNAPII) to the promoter of PRR-responsive genes ${ }^{113}$. This approach confers a net survival advantage against viral and bacterial infections as well as against sterile tissue injury in mice ${ }^{113}$, presumably through the establishment of disease tolerance. Pharmacologic use of cytokines that act in protective manner on parenchyma tissues, such as IL-10 $0^{114}$, IL-22 $2^{115}$ or EGF-like factors, such as amphiregulin ${ }^{15}$, were also used promote disease tolerance to infections in mice.

It is also possible to target pharmacologically specific resistance mechanisms associated with the development of immunopathology, as a therapeutic approach to disease tolerance to infection ${ }^{116}$. As a trade-off however, this strategy can be associated with reduced 
resistance and hence increased pathogen load, as illustrated for influenza $A$ virus ${ }^{117}$ and Trypanosoma cruzi ${ }^{118}$, as well as for human rhinoviruses ${ }^{119}$ infections in mice. In some cases, however, this does not appear to be the case, as illustrated for the therapeutic effect exerted by the cyclooxygenase inhibitor ibuprofen against Mycobacterium tuberculosis infection in mice ${ }^{116}$. These, among other studies, provide the proof-of-principle that immunoregulatory mechanisms can be targeted pharmacologically to confer tissue damage control and disease tolerance to different types of infection where anti-microbial therapy alone fails to overcome host morbidity or mortality.

Perhaps an important issue is how to develop therapeutic approaches, which might target or even identify tissue damage control mechanisms conferring disease tolerance to infection. Functional "drug screens" are one possible way forward, an approach that proved successful in identifying the ATM kinase as a molecular target via which the anthracycline [G] family of chemotherapeutic agents induce disease tolerance to sepsis in mice ${ }^{17}$. Namely, when administered at relatively low concentrations to infected mice, epirubicin, doxorubicin or daunorubicin are protective against the development of sepsis ${ }^{17}$. This therapeutic effect is associated with the induction of tissue damage control and is dissociated from the host pathogen load ${ }^{17}$. As the protective effect mediated by anthracyclines requires activation of the ATM kinase ${ }^{17}$, this indicates that targeting DNA damage responses could have potential therapeutic potential in the establishment of disease tolerance to sepsis.

Finally, a number of antibiotics are labeled as "immunomodulatory" based on their ability to improve the outcome of chronic disorders via mechanisms not readily explained on the basis of their anti-microbial activity. These fall into at least four families, namely, macrolides, fluoroquinolones, tetracyclines and polymyxines, of which macrolides have the best documented activity ${ }^{120}$. For example, the therapeutic effects of the macrolide azithromycin in chronic inflammatory pulmonary diseases including cystic fibrosis, can be dissociated from its antibacterial activity ${ }^{120}$. The "immunomodulatory" effect of these antibiotics is likely to go beyond inhibition of pro-inflammatory mediators and other immune processes and could involve the modulation of molecular pathways associated with lifespan regulation. In support of this notion, tetracyclines increase longevity in $C$. elegans via a mechanism involving the $U \mathrm{UR}^{121}$, a damage response, which as discussed above promotes disease tolerance to infections. Colistin induces the activation of the FOXO pathway, another lifespan extension pathway in C. elegans that confers disease tolerance to Gram-negative infection ${ }^{122}$. Of note, these antibiotics have not been shown to exert a direct effect on tissue damage control mechanisms and more definitive conclusions await experimental validation, for example using germ-free organisms. 


\section{CONCLUDING REMARKS AND PERSPECTIVES}

It is now clear that multicellular organisms use two genetically distinct defense strategies to limit the pathogenicity of microbes. The prevailing view has long been that immune-driven resistance mechanisms that contain, kill or expel invading microbes are the prevailing defense strategy against infectious diseases. However, disease tolerance is an equally important host defense strategy against infection, which does not exert a direct negative impact on pathogens, while interacting functionally with immune-driven resistance mechanisms to limit the severity of infectious diseases. The cellular and molecular bases of these interactions are only now starting to be appreciated. A series of experimental approaches have been used to identify and characterize the molecular and cellular basis of tissue damage control mechanisms conferring disease tolerance to a variety of pathogens. These have so far been related restricted to number of evolutionarily conserved stress and damage responses, in some cases associated with immunoregulatory responses controlling resistance mechanisms. How immunoregulatory mechanisms modulate these stress and damage responses to confer disease tolerance to infection remains largely unexplored. There is also a growing body of experimental evidence to suggest that symbiotic microbes can regulate disease tolerance as the means to prevent their pathogenicity as well as that of other pathogens. Whether this occurs via the induction of immunoregulatory mechanisms and/or the activation of stress and damage responses remains however, to be established. Deciphering the cellular and molecular nature of these interactions should be instrumental to understand and perhaps subsequently shape therapeutic strategies to manipulate protection against major infectious diseases where resistance mechanism fail to limit disease severity. Such approaches are also likely to be transformative towards overcoming the growing global health threat imposed by the emergence of multidrug resistance in pathogens as well as to treat co-infections associated with high levels of morbidity and mortality.

ACKNOWLEDGements: The authors thank Jessica Thompson and Faouzi Brazza for insightful comments and Susana Ramos for careful review and editing of the manuscript (Instituto Gulbenkian de Ciência). MPS is supported by Fundação Calouste Gulbenkian, by Fundação para a Ciência e Tecnologia (FCT; PTDC/SAU-TOX/116627/2010, HMSPICT/0022/2010) and by the European Community 7th Framework (ERC-2011-AdG 294709-DAMAGECONTROL). LFM is an FCT Investigator and is supported by the European Community Horizon 2020 (ERC-2014-CoG 647888-iPROTECTION). LT is supported by Fundação Calouste Gulbenkian. 


\section{Box 1: TOLERANCE AND DISEASE TOLERANCE.}

While there is a growing interest in understanding disease tolerance, both at a mechanistic level as well as in its potential therapeutic application, the concept and associated terminology are often misused. This is likely due to the fact that tolerance is broadly used to define some core properties of the immune system. These include immunological tolerance, which refers to an active process via which specific antigens become non-immunogenic, that is, fail to trigger adaptive immunity in a given individual ${ }^{123}$, based on immunoregulatory mechanisms that eliminate or suppress the activation and proliferation of antigen-specific $B$ and $\mathrm{T}$ cells. Immunological tolerance provides an explanation for immune self-non-self discrimination, a concept deeply rooted in the understanding of immunity. While some of the mechanisms regulating immunological tolerance and disease tolerance are functionally related these are clearly distinct phenomena.

Tolerance is also used to refer to another related phenomenon in which innate immune cells, particularly macrophages, modulate responses to bacterial lipopolysaccharide (LPS). The cellular and molecular bases for LPS tolerance have been extensively studied and relate primarily to the induction of epigenetic modifications in enhancers, which modulate gene transcription in response to TLR4 signaling ${ }^{124}$. This is not specific to TLR4, occurring downstream of other PRRs ${ }^{125}$ and other sensors ${ }^{18}$. In some instances this response, coined as "trained immunity" ${ }^{126}$, has been functionally linked to the induction of disease tolerance ${ }^{18}$.

Tolerance also refers to a related phenomenon in which exposure to a sub-lethal dose of a given agonist renders cells, tissues, organs or organisms refractory to a subsequent dosage of the same substance that would otherwise be deleterious. This adaptive response, also referred to as hormesis or accomodation relies on the activation of a number of evolutionarily conserved genetic programs that confer protection against stress and damage induced by these agonists. Some of these genetic programs overlap with those conferring tissue damage control and disease tolerance to infections ${ }^{1,3}$.

We use the term disease tolerance to refer explicitly to the same concept defined over a century ago in the plant literature ${ }^{5,6}$, in which disease tolerance defines a defense mechanism that limits "damage to functions and structures" imposed upon the host during an infection, without interfering with pathogen $\operatorname{load}^{5,6}$ (Box 1). Of note, while disease tolerance is not, by definition, associated with modulation of pathogen load, one cannot exclude its impact on pathogen physiology, not revealed by corresponding changes in pathogen load. 


\section{Box 2: IDENTIFYING MECHANISMS CONTROLLING DISEASE TOLERANCE EXPERIMENTALLY}

By definition, genetic control of disease tolerance should reveal itself by variations of disease severity without a direct correlation with pathogen load. However, while the genetic programs underlying the stress and damage responses regulating tissue damage control and disease tolerance to infection should not exert direct effects on pathogens, this is often difficult to reveal because of the inter-relationship between tissue damage control and immune-driven resistance mechanisms. For example, when operating in parenchyma cells, genes regulating tissue damage control mechanisms should allow for immune-driven resistance mechanisms to operate in a more robust and effective manner without the onset of immunopathology. As such tissue damage control mechanisms can act indirectly to exert a negative impact on pathogens. It follows that to dissociate disease tolerance from resistance to infection often requires that resistance be stably maintained, e.g. through the use of anti-microbial agents. Under such conditions, modulation of host protection from infection is likely to involve tissue damage control mechanisms that contribute to the establishment of disease tolerance. Most studies dealing with mechanisms of host protection against infection are not designed under the conceptual framework of disease tolerance and as such it is often difficult to disentangle whether host protection relies on disease tolerance versus resistance to infection.

When designing experimental approaches aimed at identifying mechanisms regulating disease tolerance one should consider the quantification of physiologic parameters reporting on host homeostasis, such as temperature, $\mathrm{O}_{2}, \mathrm{pH}$, osmolarity or glucose concentrations. These physiologic parameters however, report only indirectly on disruption of host homeostasis. This can be overcome when quantifying parameters reporting more directly on organ, e.g. brain, cardiovascular, lung, kidney or liver, function. When reported to host pathogen load these parameters can be used to reveal variations in disease tolerance. Of note, these parameters should also be quantified at steady state, i.e. before infection, defining host "vigor". When the quantification of these parameters is not possible, incidence of mortality can be used to reveal an irreversible breakdown of homeostasis ${ }^{13,16,17,20}$.

Host pathogen load should be quantified, ideally throughout the course of an infection, for example by sampling tissues where pathogens accumulate, e.g. blood in malaria, lung in pneumonia, liver in hepatitis, etc. A potential bias of this approach is that changes in pathogen behavior, such as reflected by variations in tissue tropism and accumulation are not account for. An alternative approach is to quantify pathogen load in "whole body", as performed routinely in flies ${ }^{5,9}, 10,127$. This is more challenging in other species, including rodents, but can be achieved, for example, using transgenic pathogens expressing reporter probes quantified throughout the course of an infection by whole body imaging ${ }^{29}$. 
The approaches used to reveal disease tolerance, are most often inferred form a host health reaction norm (i.e. pattern of phenotypes produced by a given genotype under different environmental conditions) to pathogen burden ${ }^{5,128}$. Variations in reaction norms, associated with changes in the slopes of their linear regressions, are also used to reveal genetic variations in disease tolerance ${ }^{5,11,128,129}$. In this reaction norm analysis, each individual (genotype) is represented according to a single health parameter and pathogen load value, i.e. ratio of minimum health parameter to maximum pathogen load ${ }^{5,11,128,129}$. As these reaction norms do not provide information on how the relation between health and pathogen load vary over time ${ }^{128}$. An alternative approach consists in plotting health parameters versus pathogen load over time ${ }^{130}$. The resulting health curves allow to follow "disease trajectories", revealed by the concomitant changes in health and pathogen load over time ${ }^{32,130}$. Under some assumptions the data from these health curves can be used to estimate individual variations in disease tolerance ${ }^{32,130}$. 
Box 3. MiCROBIOTA AND DISEASE TOLERANCE: There are numerous examples of symbiotic microorganisms that enhance resistance to infection in animals. The bacterial endosymbiont, Wolbachia, for example, enhances resistance to Drosophila C virus in flies ${ }^{10,131}$ and to dengue and chikungunya virus infections in mosquitoes ${ }^{132}$ (Figure 5). Symbiotic interactions between bacteria and mice can also promote immune-driven resistance mechanisms against viral $^{133}$, bacterial ${ }^{134}$ or protozoan ${ }^{135,136}$ infections (Figure 5). This argues that the establishment of stable symbiotic interactions between microcellular and multicellular organisms is a widespread recurrent trait that modulates host resistance against a variety of pathogens (Figure 5). Symbiotic bacteria can also modulate tissue damage control and disease tolerance to infection in multicellular organisms (Figure 5) ${ }^{22,137}$. This is again illustrated by Wolbachia, which enhances disease tolerance to flock house virus infection in flies $^{10}$. In mice, bacteria that colonize gut, such as the human symbiont Bacteroides fragilis, also confer disease tolerance to Helicobacter hepaticus infection ${ }^{138}$ while the gut $E$. coli $\mathrm{O} 21: \mathrm{H}^{+}$symbiont confers disease tolerance to Burkholderia thailadensis or $\mathrm{S}$. Typhimurium infections ${ }^{137}$. Though the mechanisms via which endosymbiotic bacteria promote disease tolerance to virus infection in insects are not clearly established, some mechanistic insight is emerging from observations of similar beneficial host-microbe interactions in mice ${ }^{22}$. For example, the gut commensal E. coli $\mathrm{O} 21: \mathrm{H}^{+}$confers disease tolerance to systemic bacterial infections via a mechanism that involves the activation of the NLRC4 inflammasome, which induces the production of IL-18 $8^{137}$. This alarmin sustains the production of insulin-like growth factor-1 (IGF-1), which prevents the development of muscle atrophy through the activation of phosphoinositide 3-kinase (PI3K)/Akt signal and the repression of the expression of the E3 ubiquitin ligases Atrogin-1 and Murf1 in muscle cells ${ }^{137}$. This confers protection against muscle and weight loss, a common pathological outcome of infection. Other mechanisms via which components of the microbiota modulate disease tolerance involve the expression of fucosylated proteins in the gut epithelia lumen, which modulate microbiota composition, metabolism and gene expression in a manner that promotes disease tolerance to enteric infection by Citrobacter rodentium ${ }^{139}$. Modulation of host metabolism by bacterial components of the microbiota ${ }^{140}$ is also likely to modulate tissue damage control mechanisms and disease tolerance to infection ${ }^{22}$.

In the same way that pathogens can modulate resistance mechanisms, some also promote disease tolerance as the means to support their own survival, proliferation and/or transmission. For example, S. Typhimurium induces the activation of host Rho GTPases and mitogen-activate protein kinases (MAPK), leading to NF-KB activation and to the expression of downstream pro-inflammatory genes in the gut epithelium. This allows $S$. typhimurium to overcome immune-driven colonization resistance imposed by resident gut microbiota 
bacteria $^{141}$, promoting gut epithelial cell invasion and systemic infection ${ }^{142}$. During epithelial invasion however, S. Typhimurium delivers, through type III secretion systems, the proteases PipA, GogA and GtgA, which target the NF-кB family members RelA and RelB and inhibit the expression of downstream pro-inflammatory genes ${ }^{143}$. This immunoregulatory effect limits tissue damage and disease severity without interfering with host pathogen load $^{143}$, revealing that this action of $S$. Typhimurium promotes disease tolerance to infection. Other examples include the induction of IL-10 by Staphylococcus aureus through a mechanism involving the recognition of bacterial peptidoglycan via TLR2 and leading to the suppression of unfettered $\mathrm{T}$ cell activation, thus conferring tissue damage control and disease tolerance to Staphylococcus aureus infection ${ }^{144}$. 


\section{Figure Legends}

Figure 1: Tissue damage control and disease tolerance. Immunity exerts a negative impact $(-\mid)$ on pathogens while triggering $(\rightarrow)$ stress and damage in host parenchyma tissues, possibly leading to cytotoxicity, tissue dysfunction and disease. Tissue damage control mechanisms involve a number of stress and damage responses that act in a concerted manner to protect parenchyma cells and tissues from damage emanating from pathogens or from immune-driven resistance mechanisms and leading to cytotoxicity, tissue dysfunction and disease. Tissue damage control mechanisms rely, initially, on stress responses that rewire metabolic pathways as the means to preserve the functional outputs of parenchyma cells $s^{1,3,145}$. If stress persists over time, damage to intracellular metabolites, macromolecules and cellular organelles develops ${ }^{1}$. This is countered by damage responses that repair these different types of damage as the means to preserve the functional outputs of parenchyma cells. If this second layer of tissue damage control fails to preserve the functional outputs of parenchyma cells, the default response becomes programmed cell death. When this occurs, the last layer of tissue damage control becomes cellular regeneration and tissue repair. If this still fails to preserve or restore the functional outputs of parenchyma tissues, the outcome is tissue dysfunction and damage, as reveled by the appearance of the clinical signs and symptoms of infectious diseases. Stress, damage and regenerative responses underlying tissue damage control are also regulated by immunity, in a manner that contributes to establish disease tolerance to infection.

Figure 2: Relative contribution of stress and damage responses to the establishment of disease tolerance to infection. Pathogen-derived toxins, such as pore-forming toxins, can act as a major driving force in the pathogenesis of infectious disease ${ }^{146}$, via the induction of host cellular stress and damage, eventually leading to programmed cell death ${ }^{146}$. This argues that toxins can impair host disease tolerance to infection via metabolic deregulation of host cells, eventually leading to programmed cell death. Production ROS and RNS by macrophages or polymorphonuclear cells is a common resistance mechanism against a variety of pathogens ${ }^{147,148}$. This however, can lead to oxidative stress ${ }^{147,148}$, which is monitored and countered by the Kelch-like ECH-associated protein 1 (KEAP1) ${ }^{28}$, which under homeostatic conditions constitutively promotes the ubiquitination and proteolytic degradation of the transcription factor NRF2 $2^{27,28}$. ROS or RNS repress KEAP1 and allow NRF2 activation, triggering the expression of effector genes regulating oxidative stress responses ${ }^{27,28}$. Hypoxia is countered by a stress response triggered by the prolyl hydroxylase domain protein $(\mathrm{PHD} 2)^{38}$. This $\mathrm{O}_{2}$ sensor constitutively represses the activity of 
the HIF family of transcription factors that under normoxic conditions ${ }^{38}$. Under hypoxia, PHD2 activity is inhibited and HIFs are activated, orchestrating the stress response to hypoxia $^{38}$. While usually associated exclusively with hypoxia, HIF activation also occurs in response to i) PRR signaling, ii) reduced intracellular iron availability or iii) inhibition of mitochondria cytochrome $\mathrm{c}$ oxidase leading to the production of ROS. Therefore HIF activation might confer metabolic adaptation to different types of stress associated with infection ${ }^{38}$. Metabolic stress, such as resulting for example from variations in intracellular ATP concentration are associated with AMP generation by adenylate kinase. This is sensed by the AMP-activated protein kinase $(A M P K)^{149}$, which triggers a metabolic stress response $^{149,150}$ likely contributing to the establishment of disease tolerance to infection ${ }^{111} 112$. Other forms of metabolic stress, such as for example resulting from variations in systemic glucose levels are sensed indirectly through blood insulin levels by the insulin receptor. This activates constitutively a signal transduction pathway involving phosphoinositide 3-kinase (PI3K) and protein kinase $\mathrm{B}(\mathrm{AKT})$ and repressing the forkhead box $\mathrm{O}$ (FOXO) family of transcription factors ${ }^{151}$. When insulin levels drop PI3K and AKT activation are reduced, promoting the activation of the FOXO family of transcription factors ${ }^{151}$. FOXO activation orchestrates a metabolic response that allows essential cellular functional outputs to be maintained under sub-optimal glucose supply ${ }^{151}$. FOXO activation in epithelial cells regulates the expression of anti-oxidant ${ }^{152}$ and antimicrobial genes ${ }^{153}$ that contribute to maintain epithelial barrier integrity, acting in a protective manner against infection in flies ${ }^{152,153}$ and mice ${ }^{153,154}$. However, activation of FOXO1 and FOXO3 $\alpha$ in muscle cells, promotes myofiber atrophy and muscle wasting associated with sepsis in mice ${ }^{155}$ and Mycobacterium marinum infection in flies ${ }^{127}$. This suggests that FOXO family members act in a tissue-specific manner to promote or repress disease tolerance to infection. Osmotic stress is sensed by the Akinase anchor protein 13 (AKAP13), which activates NFAT5 ${ }^{44}$, a transcription factor that provides metabolic adaptation to osmotic stress and tissue damage control to infection. Osmotic stress is also sensed by inflammasomes ${ }^{156}$, containing the NLRP3 or the CARD domain-containing protein $4(\mathrm{NLRC} 4)^{157}$. Metabolite damage imposed by structural modifications that promote the formation of crystals, acts in a proinflammatory manner as illustrated, for example, for uric acid-driven monosodium urate crystals ${ }^{158}$ or cholesterol crystals $^{159}$. These are sensed by NLRP3 inflammasomes, triggering a downstream signaling transduction pathway, which involves the apoptosis-associated speck-like protein containing a CARD (ASC), caspase-1 and IL-18 ${ }^{49}$. Extracellular release of metabolites, such as for example ATP, is sensed by the purinergic P2 receptor P2X ATP-gated ion channel ${ }^{160}$, which signals via inflammasomes ${ }^{156}$ to promote inflammation and tissue damage ${ }^{160}$. Catabolism of extracellular ATP/ADP into AMP and subsequently into adenosine, by the nucleoside triphosphate dephosphorylase CD39 and the ecto-5'-nucleotidase CD73, respectively ${ }^{160}$, 
exerts immunoregulatory effects, promoting the establishment of disease tolerance Toxoplasma gondii ${ }^{161}$ or Helicobacter ${ }^{162}$ infections in mice. Presumably these effects are mediated via adenosine-driven signaling through transmembrane adenosine $G$ proteincoupled cell surface receptors ${ }^{109,}{ }^{163}$. Lipid peroxidation is countered by several damage responses ${ }^{50}$ that induce $\mathrm{HO}-1^{31}$, a heme catabolizing enzyme that degrades the lipophilic pro-oxidant heme into biliverdin, which is converted by biliverdin reductase into the lipophilic anti-oxidant bilirubin ${ }^{164}$ and promotes disease tolerance to bloodstream infections ${ }^{16,20,21,29-31}$. Whether activation of the glutathione/GPX4 pathway promotes disease tolerance to infection has not been established. Proteoxic damage is sensed in the cytoplasm by chaperones from the heat shock protein (HSP) family, which activate the transcription factor heat shock factor $1(\mathrm{HSF} 1)^{54}$ and is sensed in the endoplasmic reticulum by the binding immunoglobulin protein $(\mathrm{BiP}) / 78 \mathrm{kDa}$ glucose-regulated protein (GRP-78) chaperone and the inositol requiring protein-1 (IRE1) ${ }^{53}$, which activate the unfolded protein response (UPR). HSF1 activation confers a survival advantage against Enterococcus faecalis and $P$. aeruginosa infections in Caenorhabditis elegans ${ }^{55,56}$ as well as against Listeria monocytogenes infection in mice ${ }^{57}$, where it contributes only marginally to pathogen clearance ${ }^{57}$. Activation of the UPR, via the X-box binding protein 1 (XBP1), prevents immunopathology associated with $P$. aeruginosa infection in $C$. elegans ${ }^{58}$ while preserving homeostatic control of microbiota interactions with host gut epithelia ${ }^{53}$. The DNA damage responses orchestrated by ATM ${ }^{17}$ and by $p 53^{165}$ confer tissue damage control and disease tolerance to pulmonary bacterial infections in mice. Damage to cellular organelles in countered by the autophagy response. Damage to cellular organelles is countered by autophagy, which relies on a cellular vesicular system initiated by a process of nucleation of damaged organelles ${ }^{62}$. Autophagy modulates disease tolerance to a variety of infections ${ }^{63} 6417$.

Figure 3: Immune regulation of tissue damage control. a) Tissue resident macrophages use PRR or other sensors such as interleukin receptors or AhR to sense environmental cues that alert for disruption of homeostasis, such as pathogens (green circles), dying infected cells, alarmins, e.g. DAMPs IL-1, IL-18, IL-33 or L-kynurenine generated by host parenchyma cells via the tryptophan 2,3-dioxygenase, respectively. Signaling via these sensors, polarizes macrophage responses to assist parenchyma cells in restoring homeostasis ${ }^{18,166,167}$. This non cell-autonomous mechanism supporting tissue damage control and disease tolerance to infection is mediated via the secretion of cytokines, e.g. tumor necrosis factor (TNF), IL-6, IL-10, as well as growth factors, e.g. transforming growth factor beta (TGF- $\beta$ ) and PDGF family members, among others. b) Tissue resident ILCs can also sense alarmins, e.g. IL-33, IL-1, via the corresponding IL receptors (ILR) or 
sense cellular stress and damage via natural cytotoxicity receptors $(\mathrm{NCR})^{83}$. These trigger the production of cytokines, e.g. IL-22, and EGF family members, e.g. amphiregulin (AREG), which promote tissue damage control in parenchyma cells, e.g. epithelial cells ${ }^{83,84,86}$. c) Tissue-resident $T_{\text {REG }}$ also sense alarmins, e.g. IL-18 and IL-33, which elicit the production of EGFs, i.e. amphiregulin ${ }^{89-91}$, acting on parenchyma cells to promote tissue damage control $^{89-91}$ and confer disease tolerance to infection ${ }^{90}$.

Figure 4: Pathogen class-specific tissue damage control mechanisms. a) Type 1 immunity drives resistance to viruses and intracellular bacteria (green circles), such as Listeria monocytogenes, Salmonella spp. and Mycobacteria spp., as well as against intracellular protozoan parasites such as Leishmania spp. ${ }^{99}$. Tissue damage control mechanisms countering type 1 immunopathology rely on cellular regeneration and tissue repair to restore homeostasis ${ }^{15,90}$. The mechanisms via which type 1 immunity contribute to this tissue damage control response are not clear but are likely to involve the production of EGFs, TGF- $\beta$ and PDGF. These can drive the proliferation and differentiation of stem cells into functional parenchyma cells, restoring tissue integrity and function ${ }^{23}$. b) Resistance to extracellular metazoan parasites and other large parasites is mediated and/or involves type 2 immunity ${ }^{99,100}$. Pathogen neutralization is achieved via different mechanisms orchestrated by $\mathrm{T}_{\mathrm{H}} 2$ signature cytokines, e.g. IL-4, IL-5 and IL-13, as well as by additional type 2 cytokines such as thymic stromal lymphopoietin (TSLP), IL-25 or IL-33, secreted by damaged cells ${ }^{99100} . T_{H} 2$ signature cytokines drive $B$ cell activation towards the production of high affinity pathogen-specific $\operatorname{lgG} 1$ and $\operatorname{lgE}$ antibodies that act via Fc-dependent mechanisms to trigger the activation of eosinophils, mast cells and basophils, expelling pathogens across epithelia ${ }^{100}$. Some of these parasites, e.g. helminthes, are damaging to parenchyma cells and a such type 2 immunity encompasses tissue damage control mechanisms that confer disease tolerance to infection by these parasites ${ }^{101}$. These mechanisms involve the production of EGF, VEGF, TGF- $\beta$ and resistin-like molecule $\alpha$ and $\beta$ $(R E L M \alpha / \beta)$. c) $T_{H} 17$ immunity confers resistance to extracellular bacteria such as Klebsiella pneumoniae, Escherichia coli, Citrobacter rodentium, Bordetella pertussis, Porphyromonas gingivalis, and Streptococcus pneumoniae, and also to fungi such as Candida albicans, Coccidioides posadasii, Histoplasma capsulatum and Blastomyces dermatitidis ${ }^{99}$. Activation of $T_{H} 17$ cells by cognate $T$ cell receptor (TCR)/major histocompatibility complex (MHC) class II interaction and activation of ILC3 via engagement of IL1 receptor (IL-1R) by IL-1 $\beta$ secreted from damaged cells lead to the recruitment and activation of neutrophils ${ }^{104}$. $T_{H} 17$ immunopathology is driven to a large extent by products of neutrophil activation, such as reactive oxygen species (ROS) and elastase. This is countered by tissue damage control 
mechanisms regulated directly or indirectly by IL-22, originating from $T_{H} 17, T_{H} 22$ cells (not shown) or ILC3, and promoting tissue damage control.

Figure 5. a) Symbiotic bacteria can promote resistance to infection in multicellular organisms ${ }^{22}$. Examples include the bacterial endosymbiont Wolbachia, which enhances resistance to Drosophila C virus infection in flies ${ }^{10,131}$ and to dengue or chikungunya virus infections in mosquitoes ${ }^{132,168}$. This protective effect has been linked to priming of the mosquito innate immune system and possibly competition for resources supporting pathogen replication. Symbiotic neomycin-sensitive bacteria can promote immune-driven resistance mechanisms against influenza A virus infection in mice, via mechanism involving bacterial sensing by inflammasomes ${ }^{133}$. The gut $E$. coli O86B7 commensal elicits an IgM antibody response directed against the gal $\alpha(1,3)$ gal glycan that confers resistance to Plasmodium infection in mice and possibly in humans ${ }^{135}$ while Lactobacillus and Bifidobacterium confer resistance to Plasmodium infection in mice via a mechanism that has not been clearly established ${ }^{136}$. Other gram-negative bacterial components of the mouse gut microbiota are sensed by TLR4 and trigger an antigen-specific IgG antibody responses directed against Murein lipoprotein, which confer resistance to systemic E. coli infection ${ }^{134}$. b) Symbiotic bacteria can also promote disease tolerance to infection in multicellular organisms. For example, Wolbachia enhances disease tolerance to flock house virus infection in flies ${ }^{10}$ and against Plasmodium relictum infection in mosquitoes ${ }^{169}$. Symbiotic Bacteroides fragilis induces disease tolerance to Helicobacter hepaticus infection in mice via a mechanism that involves the expression of polysaccharide $A$. This capsular glycan is sensed by the host and induces IL-10 expression ${ }^{138}$, which acts on host parenchyma cells to promote tissue damage control and disease tolerance to Helicobacter hepaticus infection ${ }^{138}$. Bacterial symbionts such Clostridium strains ${ }^{170}$ also promote the development of $T_{\text {REG }}$ cells in mice, but whether these symbiotic consortia modulate disease tolerance to infections has not been established. The gut symbiont $E$. coli $\mathrm{O} 21: \mathrm{H}^{+}$confers disease tolerance to systemic bacterial infections ${ }^{137}$ while unidentified bacterial microbiota modulate disease tolerance to enteric infection by Citrobacter rodentium ${ }^{139}$. 


\section{REFERENCES:}

1. Soares, M.P., Gozzelino, R. \& Weis, S. Tissue damage control in disease tolerance. Trends Immunol 35, 483-94 (2014).

2. Kotas, M.E. \& Medzhitov, R. Homeostasis, Inflammation, and Disease Susceptibility. Cell 160, 816-827 (2015).

3. Chovatiya, R. \& Medzhitov, R. Stress, inflammation, and defense of homeostasis. Mol Cell 54, 281-8 (2014).

References 1-3provide an overview on how different stress and damage reponses contribute to re-establishement of homeostasis after infection as well as other pathologic conditions.

4. Fauci, A.S. \& Morens, D.M. The perpetual challenge of infectious diseases. N Engl J Med 366, 454-61 (2012).

5. Schneider, D.S. \& Ayres, J.S. Two ways to survive infection: what resistance and tolerance can teach us about treating infectious diseases. Nat Rev Immunol 8, 88995 (2008).

6. Medzhitov, R., Schneider, D.S. \& Soares, M.P. Disease tolerance as a defense strategy. Science 335, 936-41 (2012).

References 5,6 provide a conceptual overview of disease tolerance to infection, bridging the initial description of disease tolerance in plants and insects to mice and possibly humans.

7. Schaefer, J.F. Tolerance to plant disease. Annual Reviews of Phytopathology 9, 235252 (1971).

8. Caldwell, R.M., Schafer, J.F., Compton, L.E. \& Patterson, F.L. Tolerance to Cereal Leaf Rusts. Science 128, 714-5 (1958).

9. $\quad$ Ayres, J.S., Freitag, N. \& Schneider, D.S. Identification of Drosophila mutants altering defense of and endurance to Listeria monocytogenes infection. Genetics 178, 180715 (2008).

10. Teixeira, L., Ferreira, A. \& Ashburner, M. The bacterial symbiont Wolbachia induces resistance to RNA viral infections in Drosophila melanogaster. PLoS Biol 6, e2 (2008).

References 9,10 are the first demonstration of disease tolerance in insects, suggesting that this defense strategy identified originally in plants can be extrapolated to animals. Reference 10 also demonstrates that host symbiotic interactions modulate disease tolerance to pathogens.

11. Raberg, L., Sim, D. \& Read, A.F. Disentangling genetic variation for resistance and tolerance to infectious diseases in animals. Science 318, 812-4 (2007).

Original demonstration and description of disease tolerance in mammals.

12. Seixas, E. et al. Heme oxygenase-1 affords protection against noncerebral forms of severe malaria. Proc Natl Acad Sci U S A 106, 15837-42 (2009).

First mechanistic study on disease tolerance in mammals, in the same experimental model used in reference 11.

13. Gozzelino, R. et al. Metabolic adaptation to tissue iron overload confers tolerance to malaria. Cell Host Microbe 12, 693-704 (2012).

14. Rodrigue-Gervais, I.G. et al. Cellular inhibitor of apoptosis protein clAP2 protects against pulmonary tissue necrosis during influenza virus infection to promote host survival. Cell Host Microbe 15, 23-35 (2014).

15. Jamieson, A.M. et al. Role of tissue protection in lethal respiratory viral-bacterial coinfection. Science 340, 1230-4 (2013).

First mechanistic demonstration that the pathological outcome of co-infections can be determined by deregulation of disease tolerance.

16. Larsen, R. et al. A central role for free heme in the pathogenesis of severe sepsis. Sci Transl Med 2, 51 ra71 (2010).

17. Figueiredo, N. et al. Anthracyclines induce DNA damage response-mediated protection against severe sepsis. Immunity 39, 874-84 (2013). 
18. Bessede, A. et al. Aryl hydrocarbon receptor control of a disease tolerance defence pathway. Nature 511, 184-90 (2014).

19. Romani, L. Immunity to fungal infections. Nat Rev Immunol 11, 275-88 (2011).

20. Ferreira, A. et al. Sickle Hemoglobin Confers Tolerance to Plasmodium Infection. Cell 145, 398-409 (2011).

21. Pamplona, A. et al. Heme oxygenase-1 and carbon monoxide suppress the pathogenesis of experimental cerebral malaria. Nat Med 13, 703-10 (2007).

22. Ayres, J.S. Cooperative Microbial Tolerance Behaviors in Host-Microbiota Mutualism. Cell 165, 1323-31 (2016).

23. Karin, M. \& Clevers, H. Reparative inflammation takes charge of tissue regeneration. Nature 529, 307-315 (2016).

24. Ayres, J.S. \& Schneider, D.S. Tolerance of infections. Annu Rev Immunol 30, 271-94 (2012).

25. Rakoff-Nahoum, S., Paglino, J., Eslami-Varzaneh, F., Edberg, S. \& Medzhitov, R. Recognition of commensal microflora by toll-like receptors is required for intestinal homeostasis. Cell 118, 229-41 (2004).

Seminal demonstration that pattern recognition receptors are involved in the maintenance of host microbiota interactions. The findings are interpreted by the authors, to suggest that signaling via PRR confers tissue damage control in the gut epithelium, which is essential for maintenance of an homeostatic balance of host microbiota interactions.

26. Ayres, J.S. Inflammasome-microbiota interplay in host physiologies. Cell Host Microbe 14, 491-7 (2013).

27. Sykiotis, G.P. \& Bohmann, D. Stress-activated cap'n'collar transcription factors in aging and human disease. Sci Signal 3, re3 (2010).

28. Hayes, J.D. \& Dinkova-Kostova, A.T. The Nrf2 regulatory network provides an interface between redox and intermediary metabolism. Trends Biochem Sci 39, 199218 (2014).

29. Jeney, V. et al. Control of disease tolerance to malaria by nitric oxide and carbon monoxide. Cell Rep 8, 126-36 (2014).

30. Ferreira, A., Balla, J., Jeney, V., Balla, G. \& Soares, M.P. A central role for free heme in the pathogenesis of severe malaria: the missing link? $J$ Mol Med 86, 1097-111 (2008).

This article proposes host labile heme generated through hemolysis acts as a central component in the pathogenesis of severe forms of malaria. The article also proposes how physiologic mechanisms that control the pathogenic effects of labile heme counter the pathogenesis of severe forms of malaria.

31. Gozzelino, R., Jeney, V. \& Soares, M.P. Mechanisms of cell protection by heme oxygenase-1. Annu Rev Pharmacol Toxicol 50, 323-54 (2010).

32. Torres, B.Y. et al. Tracking Resilience to Infections by Mapping Disease Space. PLoS Biol 14, e1002436 (2016).

33. Soares, M.P. \& Ribeiro, A.M. Nrf2 as a master regulator of tissue damage control and disease tolerance to infection. Biochem Soc Trans 43, 663-8 (2015).

34. Thimmulappa, R.K. et al. Nrf2 is a critical regulator of the innate immune response and survival during experimental sepsis. J Clin Invest 116, 984-95 (2006).

35. Athale, J. et al. Nrf2 promotes alveolar mitochondrial biogenesis and resolution of lung injury in Staphylococcus aureus pneumonia in mice. Free Radic Biol Med 53, 1584-94 (2012).

36. Kobayashi, E.H. et al. Nrf2 suppresses macrophage inflammatory response by blocking proinflammatory cytokine transcription. Nat Commun 7, 11624 (2016).

37. Eichenfield, D.Z. et al. Tissue damage drives co-localization of NF-kappaB, Smad3, and Nrf2 to direct Rev-erb sensitive wound repair in mouse macrophages. Elife $\mathbf{5}$ (2016).

38. Greer, S.N., Metcalf, J.L., Wang, Y. \& Ohh, M. The updated biology of hypoxiainducible factor. EMBO J 31, 2448-60 (2012). 
39. Cheng, S.C. et al. mTOR- and HIF-1alpha-mediated aerobic glycolysis as metabolic basis for trained immunity. Science 345, 1250684 (2014).

40. Kelly, B. \& O'Neill, L.A. Metabolic reprogramming in macrophages and dendritic cells in innate immunity. Cell Res 25, 771-84 (2015).

41. Matak, P. et al. Myeloid HIF-1 is protective in Helicobacter pylori-mediated gastritis. $J$ Immunol 194, 3259-66 (2015).

42. Wang, A. et al. Opposing Effects of Fasting Metabolism on Tissue Tolerance in Bacterial and Viral Inflammation. Cell 166, 1512-1525 e12 (2016).

43. Jantsch, J. et al. Cutaneous $\mathrm{Na}+$ storage strengthens the antimicrobial barrier function of the skin and boosts macrophage-driven host defense. Cell Metab 21, 493501 (2015).

44. Kino, T. et al. Brx mediates the response of lymphocytes to osmotic stress through the activation of NFAT5. Sci Signal 2, ra5 (2009).

45. Brocker, C., Thompson, D.C. \& Vasiliou, V. The role of hyperosmotic stress in inflammation and disease. Biomol Concepts 3, 345-364 (2012).

46. Kuper, C., Fraek, M.L., Muller, H.H., Beck, F.X. \& Neuhofer, W. Sepsis-induced urinary concentration defect is related to nitric oxide-dependent inactivation of TonEBP/NFAT5, which downregulates renal medullary solute transport proteins and aquaporin-2. Crit Care Med 40, 1887-95 (2012).

47. Qiu, Y., Hansen, P.J., Zhang, M. \& Yang, D. in Experimental Biology 2016 Meeting 920.10 (Faseb Journal, 2016).

48. Linster, C.L., Van Schaftingen, E. \& Hanson, A.D. Metabolite damage and its repair or pre-emption. Nat Chem Biol 9, 72-80 (2013).

This article proposes a new concept, namely, metabolite damage.

49. Franklin, B.S., Mangan, M.S. \& Latz, E. Crystal Formation in Inflammation. Annu Rev Immunol (2016).

This article provides a detailed conceptual framework for phase transition as a universal "danger signal" sensed by pattern recognition receptors.

50. Gutteridge, J.M. Lipid peroxidation and antioxidants as biomarkers of tissue damage. Clin Chem 41, 1819-28 (1995).

51. Imai, $Y$. et al. Identification of oxidative stress and Toll-like receptor 4 signaling as a key pathway of acute lung injury. Cell 133, 235-49 (2008).

Experimental demonstration of the extremely potent pathologic effect exerted by lipid peroxidation trough TLR4 signaling.

52. Suzuki, T., Motohashi, H. \& Yamamoto, M. Toward clinical application of the Keap1Nrf2 pathway. Trends Pharmacol Sci 34, 340-6 (2013).

53. Bettigole, S.E. \& Glimcher, L.H. Endoplasmic Reticulum Stress in Immunity. Annual Review of Immunology Vol 33 33, 107-138 (2015).

54. Lindquist, S. The heat-shock response. Annu Rev Biochem 55, 1151-91 (1986).

55. Mohri-Shiomi, A. \& Garsin, D.A. Insulin signaling and the heat shock response modulate protein homeostasis in the Caenorhabditis elegans intestine during infection. Journal of Biological Chemistry 283, 194-201 (2008).

56. Singh, V. \& Aballay, A. Heat-shock transcription factor (HSF)-1 pathway required for Caenorhabditis elegans immunity. Proc Natl Acad Sci U S A 103, 13092-7 (2006).

57. Murapa, P., Ward, M.R., Gandhapudi, S.K., Woodward, J.G. \& D'Orazio, S.E. Heat shock factor 1 protects mice from rapid death during Listeria monocytogenes infection by regulating expression of tumor necrosis factor alpha during fever. Infect Immun 79, 177-84 (2011).

58. Richardson, C.E., Kooistra, T. \& Kim, D.H. An essential role for XBP-1 in host protection against immune activation in C. elegans. Nature 463, 1092-5 (2010).

59. Filone, C.M. et al. The Master Regulator of the Cellular Stress Response (HSF1) Is Critical for Orthopoxvirus Infection. Plos Pathogens 10 (2014).

60. Weitzman, M.D. \& Weitzman, J.B. What's the damage? The impact of pathogens on pathways that maintain host genome integrity. Cell Host Microbe 15, 283-94 (2014). 
61. Shiloh, Y. \& Ziv, Y. The ATM protein kinase: regulating the cellular response to genotoxic stress, and more. Nat Rev Mol Cell Biol 14, 197-210 (2013).

62. Deretic, V., Saitoh, T. \& Akira, S. Autophagy in infection, inflammation and immunity. Nature Reviews Immunology 13, 722-737 (2013).

63. Orvedahl, A. et al. Autophagy Protects against Sindbis Virus Infection of the Central Nervous System. Cell Host \& Microbe 7, 115-127 (2010).

64. Maurer, K. et al. Autophagy Mediates Tolerance to Staphylococcus aureus AlphaToxin. Cell Host \& Microbe 17, 429-440 (2015).

65. Medzhitov, R. Origin and physiological roles of inflammation. Nature 454, 428-35 (2008).

\section{Probably the best review on inflammation, an absolute must read}

66. Pasparakis, M. \& Vandenabeele, P. Necroptosis and its role in inflammation. Nature 517, 311-20 (2015).

67. Dixon, S.J. et al. Ferroptosis: an iron-dependent form of nonapoptotic cell death. Cell 149, 1060-72 (2012).

68. Fridman, J.S. \& Lowe, S.W. Control of apoptosis by p53. Oncogene 22, 9030-9040 (2003).

69. Ashida, $\mathrm{H}$. et al. Cell death and infection: a double-edged sword for host and pathogen survival. J Cell Biol 195, 931-42 (2011).

70. Gillet, G. \& Brun, G. Viral inhibition of apoptosis. Trends in Microbiology 4, 312-317 (1996).

71. Serhan, C.N. Pro-resolving lipid mediators are leads for resolution physiology. Nature 510, 92-101 (2014).

72. Lee, T.S. \& Chau, L.Y. Heme oxygenase-1 mediates the anti-inflammatory effect of interleukin-10 in mice. Nature Medicine. 8, 240-6 (2002).

73. Chen, Y.C. et al. Nitric oxide and prostaglandin E2 participate in lipopolysaccharide/interferon-gamma-induced heme oxygenase 1 and prevent RAW264.7 macrophages from UV-irradiation-induced cell death. J Cell Biochem 86, 331-9 (2002).

74. Chen, G.Y. \& Nunez, G. Sterile inflammation: sensing and reacting to damage. Nat Rev Immunol 10, 826-37 (2010).

75. Bianchi, M.E. DAMPs, PAMPs and alarmins: all we need to know about danger. $J$ Leukoc Biol 81, 1-5 (2007).

76. Mustafa, A.K., Gadalla, M.M. \& Snyder, S.H. Signaling by gasotransmitters. Sci Signal 2, re2 (2009).

77. Wegiel, B. et al. Macrophages sense and kill bacteria through carbon monoxidedependent inflammasome activation. J Clin Invest 124, 4926-40 (2014).

This article proposes that gasotransmitters produced by macrophages are used to provide metabolic sensing of microbes and adjust resistance mechanisms accordingly.

78. Soares, M.P. \& Hamza, I. Macrophages and Iron Metabolism. Immnunity 44, 492-504 (2016).

79. Beg, A.A. \& Baltimore, D. An essential role for NF-kB in preventing TNF-a-induced cell death. Science 274, 782-784 (1996).

80. Buchon, N., Broderick, N.A., Poidevin, M., Pradervand, S. \& Lemaitre, B. Drosophila intestinal response to bacterial infection: activation of host defense and stem cell proliferation. Cell Host Microbe 5, 200-11 (2009).

Original finding revealing a critical role for regenerative responses of the gut epithelium as a defence strategy against enteric pathogens.

81. Hochmuth, C.E., Biteau, B., Bohmann, D. \& Jasper, H. Redox regulation by Keap1 and Nrf2 controls intestinal stem cell proliferation in Drosophila. Cell Stem Cell 8, 188-99 (2011).

82. Bonfini, A., Liu, X. \& Buchon, N. From pathogens to microbiota: How Drosophila intestinal stem cells react to gut microbes. Dev Comp Immunol (2016). 
83. Artis, D. \& Spits, H. The biology of innate lymphoid cells. Nature 517, 293-301 (2015).

84. Monticelli, L.A. et al. Innate lymphoid cells promote lung-tissue homeostasis after infection with influenza virus. Nat Immunol 12, 1045-54 (2011).

85. Turner, J.E. et al. IL-9-mediated survival of type 2 innate lymphoid cells promotes damage control in helminth-induced lung inflammation. Journal of Experimental Medicine 210, 2951-2965 (2013).

86. Monticelli, L.A. et al. IL-33 promotes an innate immune pathway of intestinal tissue protection dependent on amphiregulin-EGFR interactions. Proceedings of the National Academy of Sciences of the United States of America 112, 10762-10767 (2015).

87. Lindemans, C.A. et al. Interleukin-22 promotes intestinal-stem-cell-mediated epithelial regeneration. Nature 528, 560-+ (2015).

88. Josefowicz, S.Z., Lu, L.F. \& Rudensky, A.Y. Regulatory T cells: mechanisms of differentiation and function. Annu Rev Immunol 30, 531-64 (2012).

89. Burzyn, D. et al. A special population of regulatory $T$ cells potentiates muscle repair. Cell 155, 1282-95 (2013).

90. Arpaia, N. et al. A Distinct Function of Regulatory T Cells in Tissue Protection. Cell 162, 1078-89 (2015).

91. Schiering, C. et al. The alarmin IL-33 promotes regulatory T-cell function in the intestine. Nature 513, 564-8 (2014).

Articles 89-90 are the original description of a unsuspected physiologic role for TREG, namely in conferring tissue damage control and disease tolerance to infections (90).

92. Noel, S. et al. T Lymphocyte-Specific Activation of Nrf2 Protects from AKI. J Am Soc Nephrol 26, 2989-3000 (2015).

93. Cramer, T. et al. HIF-1alpha is essential for myeloid cell-mediated inflammation. Cell 112, 645-57 (2003).

94. Dang, E.V. et al. Control of $\mathrm{T}(\mathrm{H}) 17 / \mathrm{T}(\mathrm{reg})$ balance by hypoxia-inducible factor 1. Cell 146, 772-84 (2011).

95. McFall-Ngai, M. et al. Animals in a bacterial world, a new imperative for the life sciences. Proc Natl Acad Sci U S A 110, 3229-36 (2013).

96. Ayres, J.S. \& Schneider, D.S. The role of anorexia in resistance and tolerance to infections in Drosophila. PLoS Biol 7, e1000150 (2009).

97. Jamieson, A.M., Yu, S., Annicelli, C.H. \& Medzhitov, R. Influenza virus-induced glucocorticoids compromise innate host defense against a secondary bacterial infection. Cell Host Microbe 7, 103-14 (2010).

98. Cunnington, A.J., de Souza, J.B., Walther, M. \& Riley, E.M. Malaria impairs resistance to Salmonella through heme- and heme oxygenase-dependent dysfunctional granulocyte mobilization. Nat Med 18, 120-7 (2012).

Articles 97 and 98 are the original findings that disease tolerance to one pathogen class can compromise immune driven resistance to co-infections by another pathogen class.

99. Kara, E.E. et al. Tailored Immune Responses: Novel Effector Helper T Cell Subsets in Protective Immunity. Plos Pathogens 10 (2014).

100. Wynn, T.A. Type 2 cytokines: mechanisms and therapeutic strategies. Nat Rev Immunol 15, 271-82 (2015).

101. Allen, J.E. \& Wynn, T.A. Evolution of Th2 immunity: a rapid repair response to tissue destructive pathogens. PLoS Pathog 7, e1002003 (2011).

102. Soares, M.P. \& Weiss, G. The Iron age of host-microbe interactions. EMBO Rep 16, 1482-500 (2015).

103. Murray, P.J. Amino acid auxotrophy as a system of immunological control nodes. Nature Immunology 17, 132-139 (2016). 
104. Sahoo, M., Del Barrio, L., Miller, M.A. \& Re, F. Neutrophil elastase causes tissue damage that decreases host tolerance to lung infection with burkholderia species. PLoS Pathog 10, e1004327 (2014).

105. Gimblet, C. et al. IL-22 Protects against Tissue Damage during Cutaneous Leishmaniasis. Plos One 10 (2015).

106. Song, X. et al. Growth Factor FGF2 Cooperates with Interleukin-17 to Repair Intestinal Epithelial Damage. Immunity 43, 488-501 (2015).

107. Robinson, K.M., Kolls, J.K. \& Alcorn, J.F. The immunology of influenza virusassociated bacterial pneumonia. Curr Opin Immunol 34, 59-67 (2015).

108. Castiglia, V. et al. Type I Interferon Signaling Prevents IL-1 $\beta$-Driven Lethal Systemic Hyperinflammation during Invasive Bacterial Infection of Soft Tissue. . Cell Host \& Microbe 19, 375-387 (2016).

109. Sullivan, G.W., Fang, G., Linden, J. \& Scheld, W.M. A2A adenosine receptor activation improves survival in mouse models of endotoxemia and sepsis. J Infect Dis 189, 1897-904 (2004).

110. Brouard, S. et al. Carbon monoxide generated by heme oxygenase 1 suppresses endothelial cell apoptosis. J Exp Med 192, 1015-26 (2000).

Original description of the cytoprotective effects of the gasotransmitter carbon monoxide.

111. Escobar, D.A. et al. Adenosine monophosphate-activated protein kinase activation protects against sepsis-induced organ injury and inflammation. J Surg Res 194, 26272 (2015).

112. Mulchandani, N. et al. Stimulation of Brain AMP-Activated Protein Kinase Attenuates Inflammation and Acute Lung Injury in Sepsis. Mol Med 21, 637-44 (2015).

113. Rialdi, A. et al. Topoisomerase 1 inhibition suppresses inflammatory genes and protects from death by inflammation. Science 352, aad7993 (2016).

114. Couper, K.N., Blount, D.G. \& Riley, E.M. IL-10: the master regulator of immunity to infection. J Immunol 180, 5771-7 (2008).

115. Muhl, H. et al. IL-22 in tissue-protective therapy. Br J Pharmacol 169, 761-71 (2013).

116. Vilaplana, C. et al. Ibuprofen therapy resulted in significantly decreased tissue bacillary loads and increased survival in a new murine experimental model of active tuberculosis. J Infect Dis 208, 199-202 (2013).

117. Carey, M.A. et al. Contrasting effects of cyclooxygenase-1 (COX-1) and COX-2 deficiency on the host response to influenza A viral infection. J Immunol 175, 687884 (2005).

118. Hideko Tatakihara, V.L. et al. Effects of cyclooxygenase inhibitors on parasite burden, anemia and oxidative stress in murine Trypanosoma cruzi infection. FEMS Immunol Med Microbiol 52, 47-58 (2008).

119. Stanley, E.D., Jackson, G.G., Panusarn, C., Rubenis, M. \& Dirda, V. Increased virus shedding with aspirin treatment of rhinovirus infection. JAMA 231, 1248-51 (1975).

120. Tauber, S.C. \& Nau, R. Immunomodulatory properties of antibiotics. Curr Mol Pharmacol 1, 68-79 (2008).

121. Houtkooper, R.H. et al. Mitonuclear protein imbalance as a conserved longevity mechanism. Nature 497, 451-7 (2013).

122. Cai, Y., Cao, X. \& Aballay, A. Whole-animal chemical screen identifies colistin as a new immunomodulator that targets conserved pathways. MBio 5 (2014).

123. Billingham, R.E., Brent, L. \& Medawar, P.B. Actively acquired tolerance of foreign cells. Nature 172, 603-6 (1953).

124. Foster, S.L., Hargreaves, D.C. \& Medzhitov, R. Gene-specific control of inflammation by TLR-induced chromatin modifications. Nature 447, 972-8 (2007).

125. Saeed, S. et al. Epigenetic programming of monocyte-to-macrophage differentiation and trained innate immunity. Science 345, 1251086 (2014).

126. Netea, M.G., Latz, E., Mills, K.H. \& O'Neill, L.A. Innate immune memory: a paradigm shift in understanding host defense. Nat Immunol 16, 675-9 (2015). 
Original findings on a mechanistic basis for LPS tolerance in macrophages (124), expanded thereafter to other PRR agonists and coined as "trained immunity" $(125,126)$.

127. Dionne, M.S., Pham, L.N., Shirasu-Hiza, M. \& Schneider, D.S. Akt and FOXO dysregulation contribute to infection-induced wasting in Drosophila. Curr Biol 16, 1977-85 (2006).

128. Simms, E.L. Defining tolerance as a norm of reaction. Evolutionary Ecology 14, 563570 (2000).

129. Raberg, L., Graham, A.L. \& Read, A.F. Decomposing health: tolerance and resistance to parasites in animals. Philos Trans $R$ Soc Lond B Biol Sci 364, 37-49 (2009).

130. Schneider, D.S. Tracing personalized health curves during infections. PLoS Biol 9, e1001158 (2011).

Original description of disease curves and their interpretation as a novel way to understand host pathogen interactions.

131. Hedges, L.M., Brownlie, J.C., O'Neill, S.L. \& Johnson, K.N. Wolbachia and virus protection in insects. Science 322, 702 (2008).

132. Moreira, L.A. et al. A Wolbachia symbiont in Aedes aegypti limits infection with dengue, Chikungunya, and Plasmodium. Cell 139, 1268-78 (2009).

133. Ichinohe, $\mathrm{T}$. et al. Microbiota regulates immune defense against respiratory tract influenza A virus infection. Proc Natl Acad Sci U S A 108, 5354-9 (2011).

134. Zeng, Melody Y. et al. Gut Microbiota-Induced Immunoglobulin G Controls Systemic Infection by Symbiotic Bacteria and Pathogens. Immunity 44, 647-658 (2016).

135. Yilmaz, B. et al. Gut Microbiota Elicits a Protective Immune Response against Malaria Transmission. Cell 159, 1277-89 (2014).

136. Villarino, N.F. et al. Composition of the gut microbiota modulates the severity of malaria. Proc Natl Acad Sci U S A (2016).

137. Schieber, A.M. et al. Disease tolerance mediated by microbiome E. coli involves inflammasome and IGF-1 signaling. Science 350, 558-63 (2015).

Articles 135-137 provide mechanistic demonstrations of how symbiotic interactions with different bacteria in the microbiota modulate resistance $(135,136)$ or disease tolerance $(137)$ to infections by blood borne pathogens.

138. Mazmanian, S.K., Round, J.L. \& Kasper, D.L. A microbial symbiosis factor prevents intestinal inflammatory disease. Nature 453, 620-5 (2008).

139. Pickard, J.M. et al. Rapid fucosylation of intestinal epithelium sustains hostcommensal symbiosis in sickness. Nature 514, 638-41 (2014).

140. Brestoff, J.R. \& Artis, D. Commensal bacteria at the interface of host metabolism and the immune system. Nat Immunol 14, 676-84 (2013).

141. Buffie, C.G. \& Pamer, E.G. Microbiota-mediated colonization resistance against intestinal pathogens. Nat Rev Immunol 13, 790-801 (2013).

142. Stecher, B. et al. Salmonella enterica serovar typhimurium exploits inflammation to compete with the intestinal microbiota. PLoS Biol 5, 2177-89 (2007).

143. Sun, H., Kamanova, J., Lara-Tejero, M. \& Galan, J.E. A Family of Salmonella Type III Secretion Effector Proteins Selectively Targets the NF-kappaB Signaling Pathway to Preserve Host Homeostasis. PLoS Pathog 12, e1005484 (2016).

144. Chau, T.A. et al. Toll-like receptor 2 ligands on the staphylococcal cell wall downregulate superantigen-induced $\mathrm{T}$ cell activation and prevent toxic shock syndrome. Nat Med 15, 641-8 (2009).

145. Naquet, P., Giessner, C. \& Galland, F. Metabolic adaptation of tissues to stress releases metabolites influencing innate immunity. Current Opinion in Immunology 38, 30-38 (2016).

146. Dal Peraro, M. \& van der Goot, F.G. Pore-forming toxins: ancient, but never really out of fashion. Nat Rev Microbiol 14, 77-92 (2016). 
147. Nathan, C. \& Shiloh, M.U. Reactive oxygen and nitrogen intermediates in the relationship between mammalian hosts and microbial pathogens. Proc Natl Acad Sci U S A 97, 8841-8 (2000).

148. Beckman, J.S. \& Koppenol, W.H. Nitric oxide, superoxide, and peroxynitrite: the good, the bad, and ugly. Am J Physiol 271, C1424-37 (1996).

149. Hardie, D.G., Ross, F.A. \& Hawley, S.A. AMPK: a nutrient and energy sensor that maintains energy homeostasis. Nat Rev Mol Cell Biol 13, 251-62 (2012).

150. O'Neill, L.A. \& Hardie, D.G. Metabolism of inflammation limited by AMPK and pseudo-starvation. Nature 493, 346-55 (2013).

151. Eijkelenboom, A. \& Burgering, B.M. FOXOs: signalling integrators for homeostasis maintenance. Nat Rev Mol Cell Biol 14, 83-97 (2013).

152. Ahn, H.M., Lee, K.S., Lee, D.S. \& Yu, K. JNK/FOXO mediated PeroxiredoxinV expression regulates redox homeostasis during Drosophila melanogaster gut infection. Dev Comp Immunol 38, 466-73 (2012).

153. Becker, T. et al. FOXO-dependent regulation of innate immune homeostasis. Nature 463, 369-73 (2010).

154. Seiler, F. et al. FOXO transcription factors regulate innate immune mechanisms in respiratory epithelial cells. J Immunol 190, 1603-13 (2013).

155. Reed, S.A., Sandesara, P.B., Senf, S.M. \& Judge, A.R. Inhibition of FoxO transcriptional activity prevents muscle fiber atrophy during cachexia and induces hypertrophy. FASEB J 26, 987-1000 (2012).

156. Latz, E., Xiao, T.S. \& Stutz, A. Activation and regulation of the inflammasomes. Nat Rev Immunol 13, 397-411 (2013).

157. Ip, W.K. \& Medzhitov, R. Macrophages monitor tissue osmolarity and induce inflammatory response through NLRP3 and NLRC4 inflammasome activation. Nat Commun 6, 6931 (2015).

158. Martinon, F., Petrilli, V., Mayor, A., Tardivel, A. \& Tschopp, J. Gout-associated uric acid crystals activate the NALP3 inflammasome. Nature 440, 237-41 (2006).

159. Duewell, P. et al. NLRP3 inflammasomes are required for atherogenesis and activated by cholesterol crystals. Nature 464, 1357-61 (2010).

160. Idzko, M., Ferrari, D. \& Eltzschig, H.K. Nucleotide signalling during inflammation. Nature 509, 310-317 (2014).

161. Mahamed, D.A., Toussaint, L.E. \& Bynoe, M.S. CD73-generated adenosine is critical for immune regulation during Toxoplasma gondii infection. Infect Immun 83, 721-9 (2015).

162. Alam, M.S. et al. CD73 is expressed by human regulatory $T$ helper cells and suppresses proinflammatory cytokine production and Helicobacter felis-induced gastritis in mice. J Infect Dis 199, 494-504 (2009).

163. Ohta, A. \& Sitkovsky, M. Role of G-protein-coupled adenosine receptors in downregulation of inflammation and protection from tissue damage. Nature 414, 91620 (2001).

164. Stocker, R., Yamamoto, Y., McDonagh, A.F., Glazer, A.N. \& Ames, B.N. Bilirubin is an antioxidant of possible physiological importance. Science 235, 1043-6 (1987).

165. Madenspacher, J.H. et al. p53 Integrates host defense and cell fate during bacterial pneumonia. J Exp Med 210, 891-904 (2013).

166. Okabe, Y. \& Medzhitov, R. Tissue biology perspective on macrophages. Nat Immunol 17, 9-17 (2015).

167. Davies, L.C., Jenkins, S.J., Allen, J.E. \& Taylor, P.R. Tissue-resident macrophages. Nat Immunol 14, 986-95 (2013).

168. Dutra, H.L. et al. Wolbachia Blocks Currently Circulating Zika Virus Isolates in Brazilian Aedes aegypti Mosquitoes. Cell Host Microbe 19, 771-4 (2016).

169. Zele, F., Nicot, A., Duron, O. \& Rivero, A. Infection with Wolbachia protects mosquitoes against Plasmodium-induced mortality in a natural system. $J$ Evol Biol 25, 1243-52 (2012). 
170. Atarashi, K. et al. Induction of colonic regulatory $\mathrm{T}$ cells by indigenous Clostridium species. Science 331, 337-41 (2011). 


\section{GLOSSARY}

Homeostasis: Maintenance of a stable physiologic "internal" state in multicellular organisms via feedback mechanisms that allow physiologic functions to proceed despite variations in the "external" environment.

Immunopathology: Refers to a breakdown of homeostasis in which immunity acts as the main cause of disease.

Necroptosis: A specific form of programmed cell death mediated via a genetically encoded mechanism involving the receptor-interacting serine/threonine-protein kinases 1 and 3 (RIPK1/3) and the mixed lineage kinase domain-like (MLKL) pseudokinase.

Ferroptosis: Genetically encoded form of programmed cell death driven by loss of activity of the lipid repair enzyme glutathione peroxidase 4 (GPX4) and the accumulation of lipid hydroperoxides.

Nutritional immunity: An evolutionary conserved resistance mechanisms against infection based on the host's ability to withhold nutrients, such as iron, from pathogens.

Alarmins: Endogenous molecules released from damaged cells and sensed by receptors of the immune system that alert for tissue dysfunction or damage, associated with disruption of homeostasis.

Anthracycline: A class of red aromatic polyketides drugs derived from Streptomyces bacteria that intercalate into DNA, arresting transcription and cell division, a property widely used therapeutically against cancers.

Stress: Any variations in the "external" environment that disrupts the maintenance of a stable physiologic environment where biologic processes are allowed to proceed. 


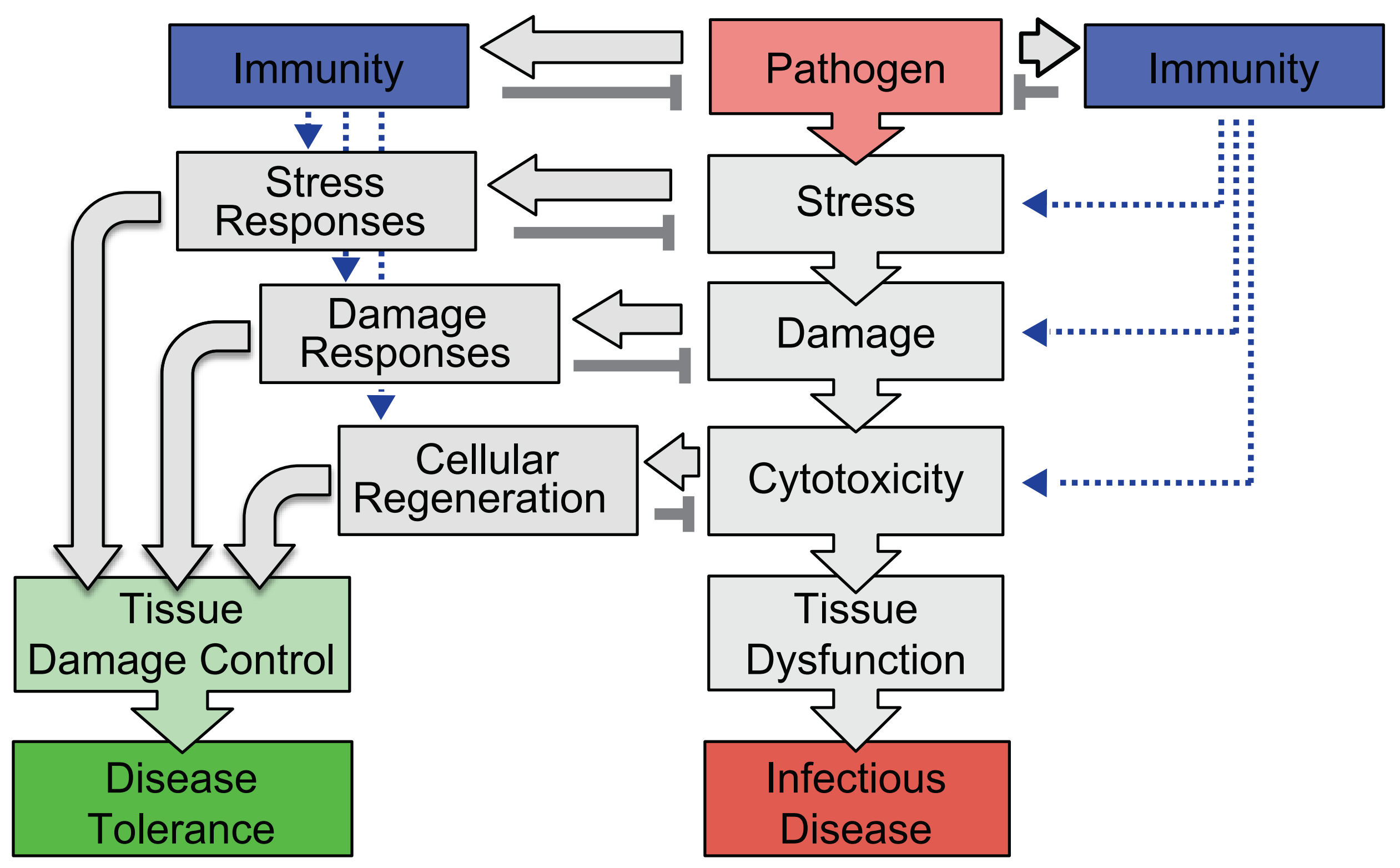




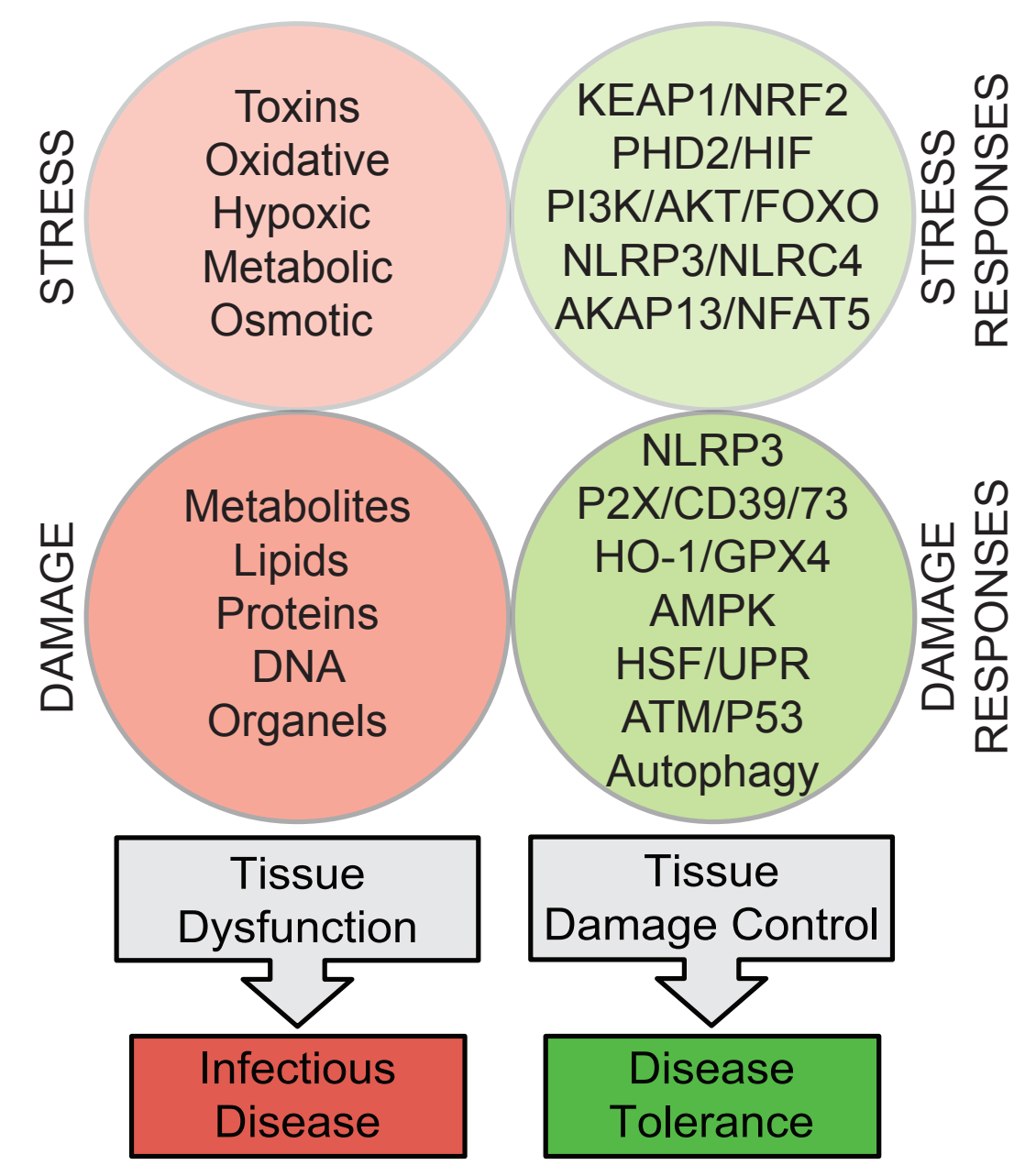


a

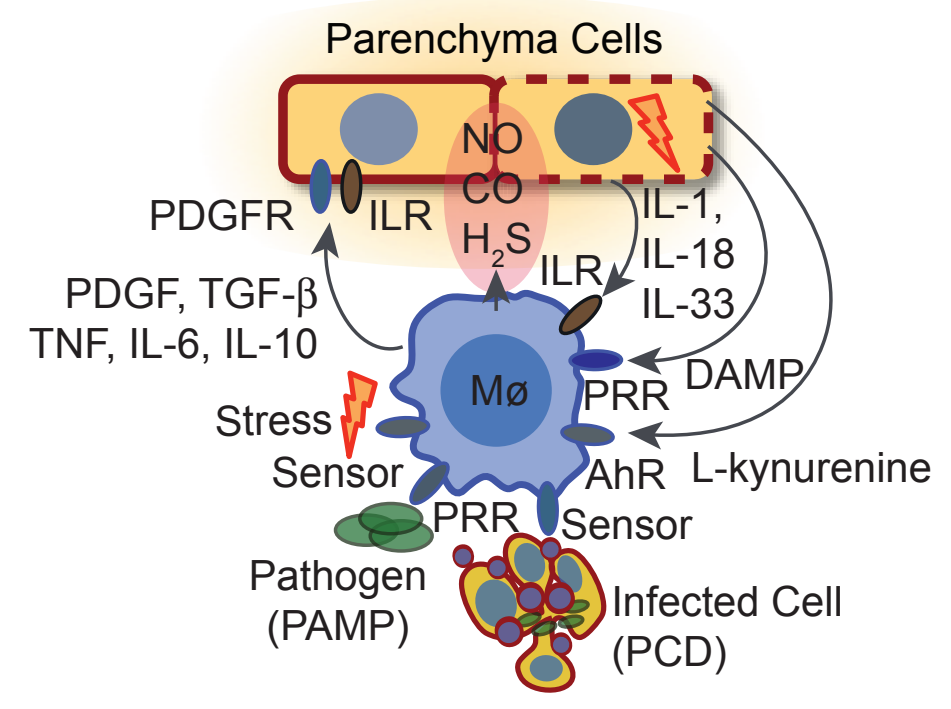

$b$

C

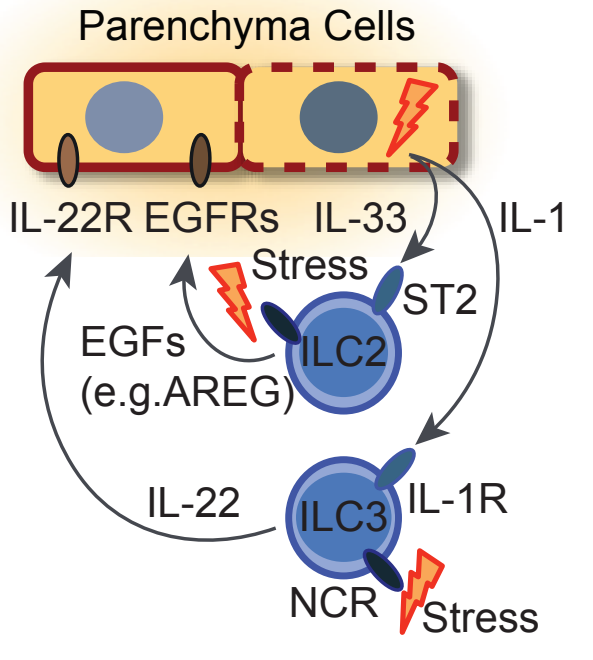

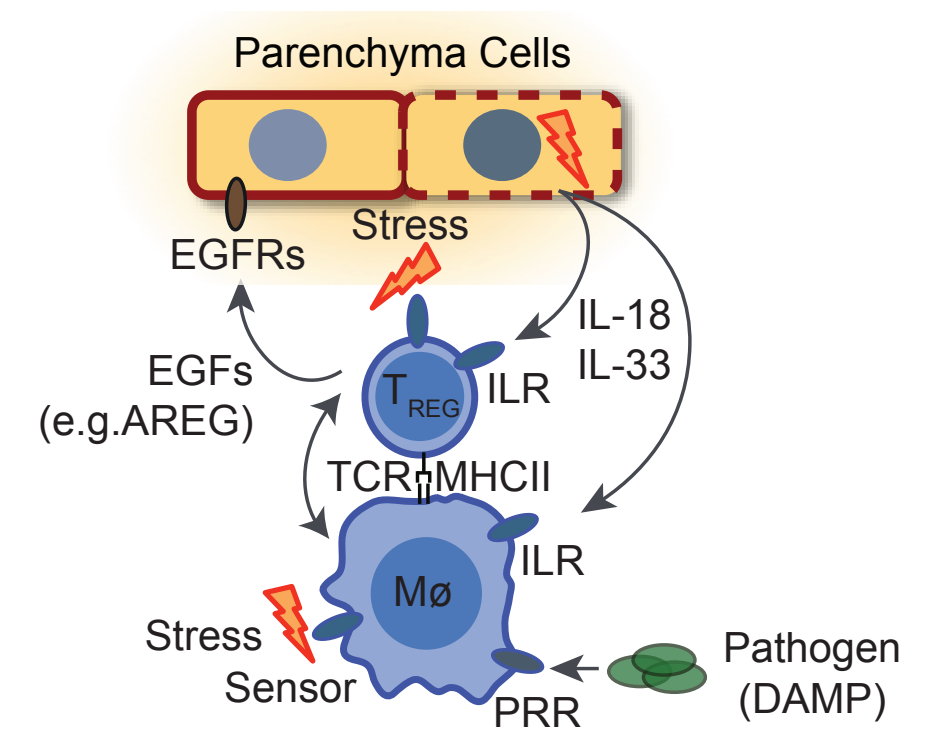


Figure 4. Disease Tolerance and Immunity Soares, M.P., Teixeira, L. and Moita L.F.

a

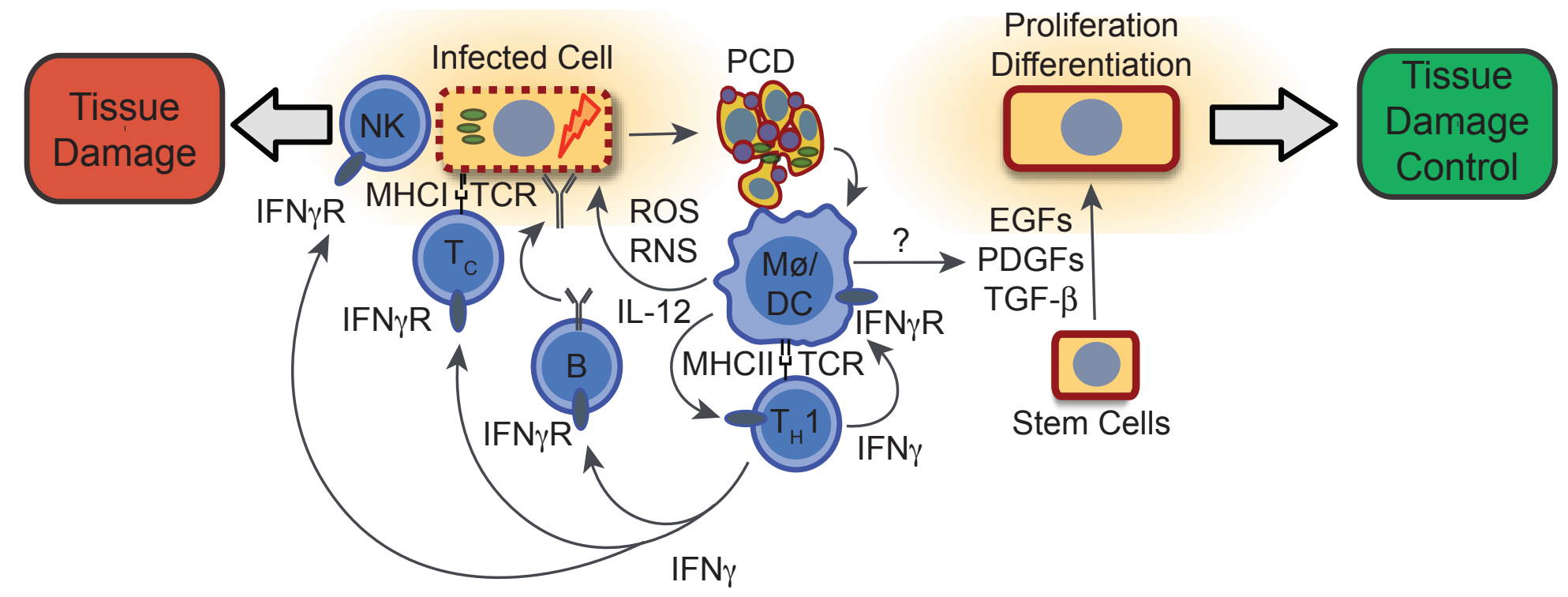

$b$

TSLP

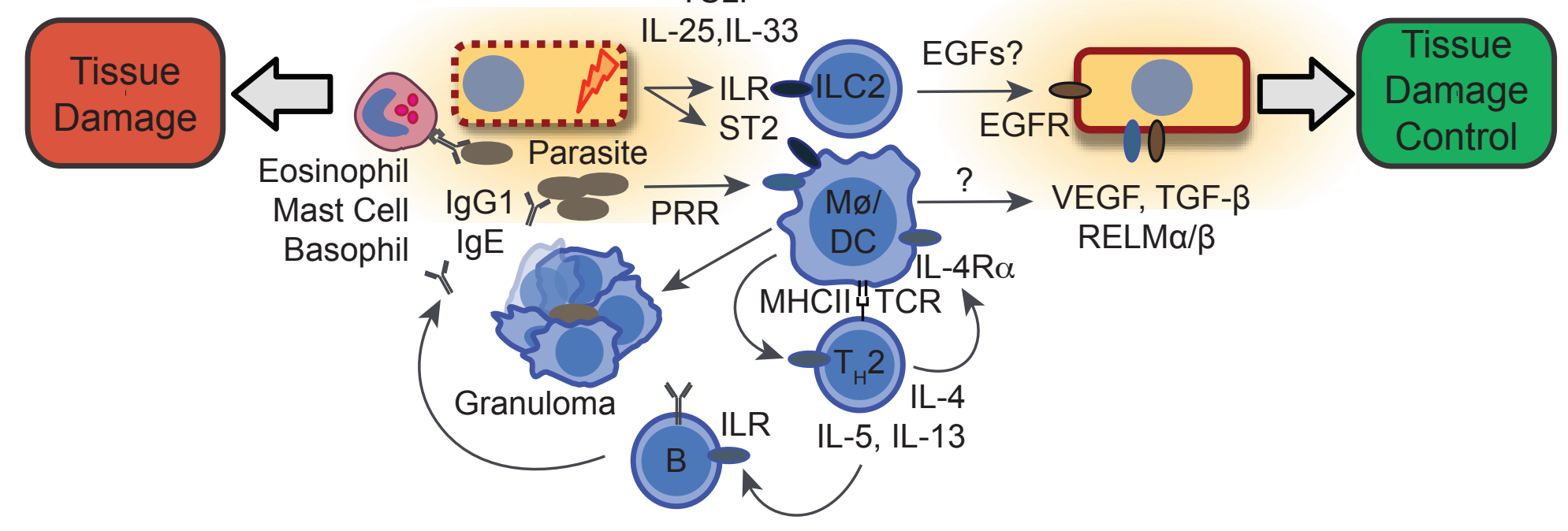

C

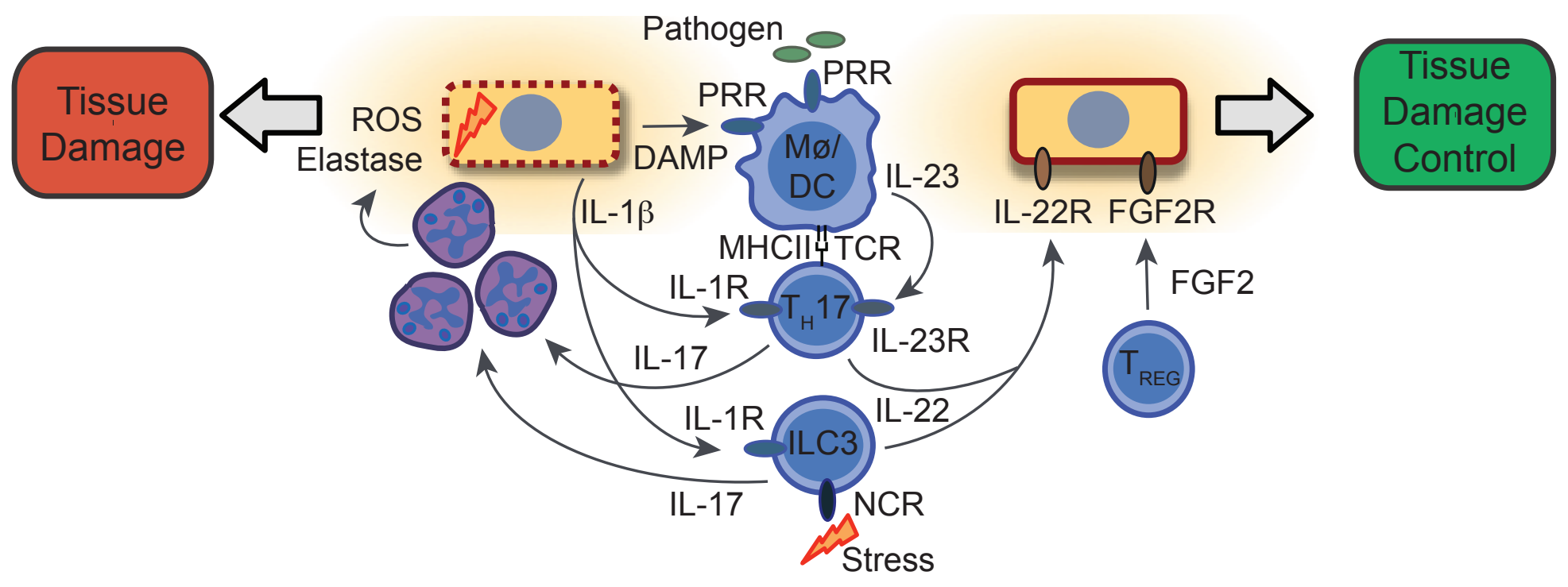


Resistance to:

Drosophila $C$ virus,

Dengue virus, chikungunya virus

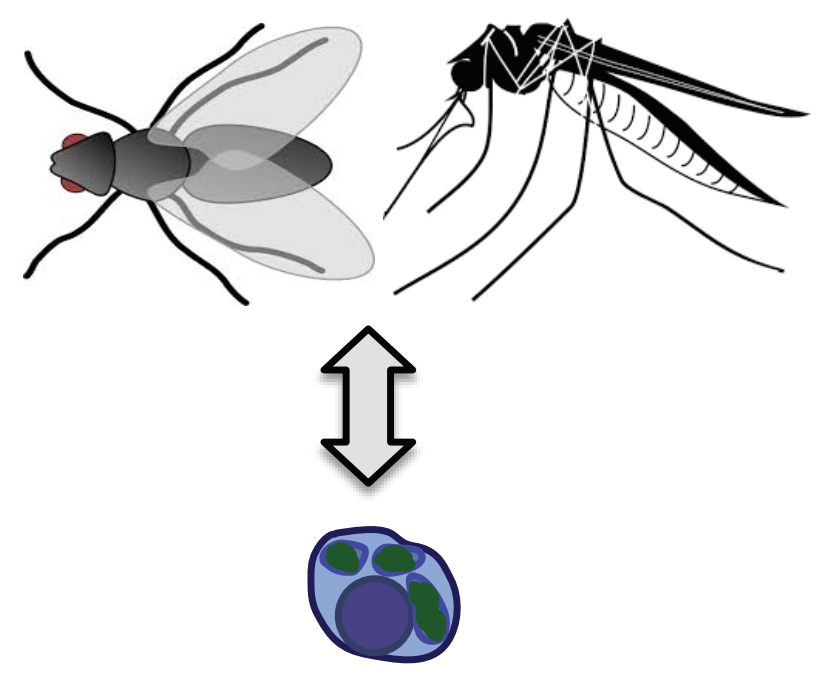

Endosymbiotic

Bacteria: Wolbachia
Resistance to:

Influenza A virus; E. coli, Plasmodium

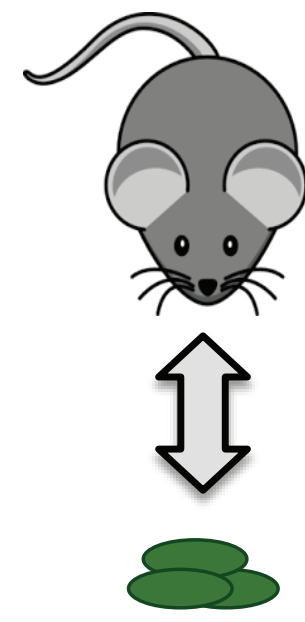

Symbiotic Bacteria:

Microbiota

$b$

Disease Tolerance to:

Flock House virus,

Plasmodium relictum
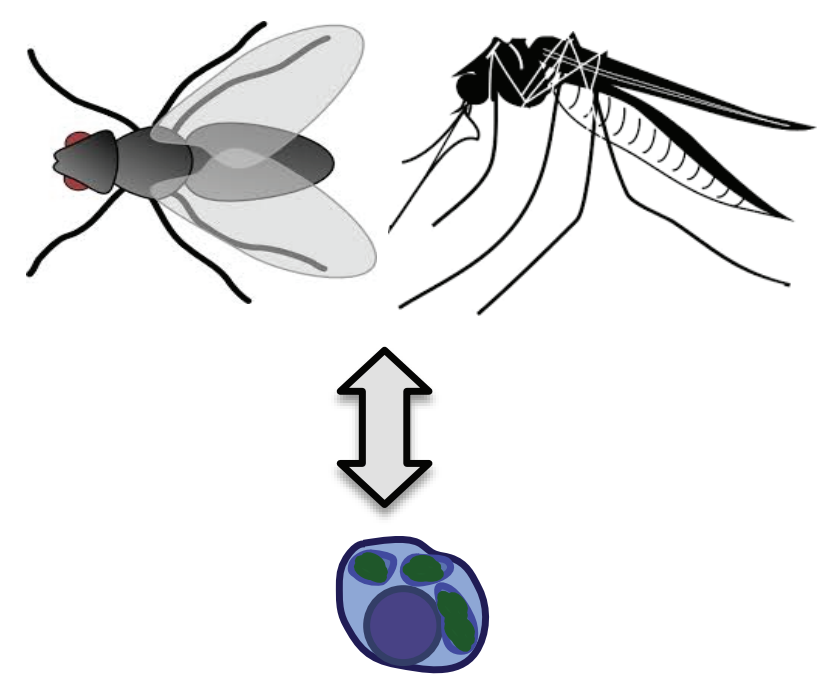

Endosymbiotic

Bacteria: Wolbachia
Disease Tolerance to:

Helicobacter hepaticus,

Burkholderia thailadensis

Salmonella, Citrobacter rodentium

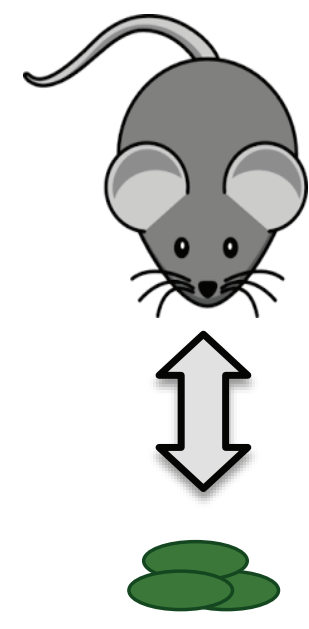

Symbiotic Bacteria: Bacteroides fragilis E. coli O21:H+ 